TRANSACTIONS OF THE

AMERICAN MATHEMATICAL SOCIETY

Volume 364, Number 6, June 2012, Pages 3265-3288

S 0002-9947(2011)05545-5

Article electronically published on November 7, 2011

\title{
INVERSE SPECTRAL PROBLEM WITH PARTIAL INFORMATION GIVEN ON THE POTENTIAL AND NORMING CONSTANTS
}

\author{
GUANGSHENG WEI AND HONG-KUN XU
}

\begin{abstract}
The inverse spectral problem for a Sturm-Liouville equation in Liouville form with separated self-adjoint boundary conditions on the unit interval $[0,1]$ is considered. Some uniqueness results are obtained which imply that the potential $q$ can be completely determined even if only partial information is given on $q$ together with partial information on the spectral data, consisting of either one full spectrum and a subset of norming constants or a subset of pairs of eigenvalues and the corresponding norming constants. Moreover, the problem of missing eigenvalues and norming constants is also investigated in the situation where the potential $q$ is $C^{2 k-1}$ and the boundary conditions at the endpoints 0 and 1 are fixed.
\end{abstract}

\section{INTRODUCTION}

We are concerned with an inverse problem for the Sturm-Liouville equation. Consider the operator $L:=L\left(q, h_{0}, h_{1}\right)$ defined by

$$
L u=-u^{\prime \prime}+q u
$$

on $[0,1]$ with the boundary conditions

$$
\begin{aligned}
& u^{\prime}(0)-h_{0} u(0)=0, \\
& u^{\prime}(1)-h_{1} u(1)=0 .
\end{aligned}
$$

Here the potential $q \in L^{1}[0,1]$ is real-valued and $h_{0}, h_{1} \in \overline{\mathbb{R}}:=\mathbb{R} \cup\{\infty\}$. Note that, in an obvious notation, $h_{0}=\infty$ singles out the Dirichlet boundary condition $u(0)=0$.

It is well known 36 that the operator $L$ subject to the boundary conditions (1.2) and (1.3) is self-adjoint and bounded below in the space $L^{2}[0,1]$ and that it has a discrete spectrum consisting of simple real eigenvalues. We use $\sigma(L)$ to denote this spectrum; thus we can write

$$
\sigma(L)=\left\{\lambda_{j}: j \in \mathbb{N}_{0}:=\{0\} \cup \mathbb{N}=\{0,1,2, \ldots\}\right\} .
$$

For problems (1.1)-(1.3) there are three main sets of spectral data which may be given to determine a unique triple $\left(q, h_{0}, h_{1}\right)$, and each of which includes the spectrum $\sigma(L)$.

Received by the editors August 9, 2010 and, in revised form, January 15, 2011.

2010 Mathematics Subject Classification. Primary 34A55; Secondary 34L40, 34L20.

Key words and phrases. Eigenvalue, norming constant, boundary condition, inverse spectral problem.

(C)2011 American Mathematical Society Reverts to public domain 28 years from publication 
If $q$ is symmetric about $x=1 / 2$ and $h_{0}=-h_{1}$, then $\sigma(L)$ alone is sufficient to recover $q$ uniquely (see [17, 19]). However, in general, an additional infinite sequence of spectral data must be given for the unique determination of $\left(q, h_{0}, h_{1}\right)$. Three different possibilities for these data include:

(i) a second set of eigenvalues $\sigma(\tilde{L})$, where (1.1) and (1.2) remain the same but where in $(1.3) h_{1}$ is replaced by some $\widetilde{h}_{1} \neq h_{1}$;

(ii) the set of norming constants $\alpha_{n}=\left\|v_{n}\right\|^{2} /\left|v_{n}(1)\right|^{2}, n \in \mathbb{N}_{0}$, when $h_{1} \in \mathbb{R}$ or $\alpha_{n}=\left\|v_{n}\right\|^{2} /\left|v_{n}^{\prime}(1)\right|^{2}, n \in \mathbb{N}_{0}$, when $h_{1}=\infty$, where $v_{n}=v\left(x, \lambda_{n}\right)$ is the eigenfunction of problems (1.1)-(1.3) corresponding to the eigenvalue $\lambda_{n}$ and $\left\|v_{n}\right\|$ is the $L^{2}$-norm of $v_{n}$;

(iii) the set of ratios $\kappa_{n}=v_{n}(0) / v_{n}(1)$ when $h_{1} \in \mathbb{R}$ or $\kappa_{n}=v_{n}^{\prime}(0) / v_{n}^{\prime}(1)$ when $h_{1}=\infty$.

Note that in some literature the ratios $\kappa_{n}$ may be written as the form of $\ln \left|v_{n}(0) / v_{n}(1)\right|$ if $h_{1} \in \mathbb{R}$ or $\ln \left|v_{n}^{\prime}(0) / v_{n}^{\prime}(1)\right|$ if $h_{1}=\infty$, which are called the terminal velocities or norming constants; see [6, 7, 8, 21, 22, 32. Indeed, based on the following relation between $\kappa_{n}$ and $\alpha_{n}$,

$$
\kappa_{n} \alpha_{n}=\omega^{\prime}\left(\lambda_{n}\right)
$$

where $\omega(\cdot)$ is the characteristic function of $L$ defined by (3.6) below (see [12, p. 18; [26]), the pair of sequences $\Gamma_{1}:=\left\{\lambda_{n}, \alpha_{n} ; n \in \mathbb{N}_{0}\right\}$ is equivalent to the pair of sequences $\Gamma_{2}:=\left\{\lambda_{n}, \kappa_{n} ; n \in \mathbb{N}_{0}\right\}$. It is also known [26] that knowing the eigenvalues $\left\{\lambda_{n}\right\}_{n \in \mathbb{N}_{0}}$ and the norming constants $\left\{\alpha_{n}\right\}_{n \in \mathbb{N}_{0}}$ is equivalent to knowing a singular measure defined by the spectral function for problems (1.1)-(1.3). Moreover, the norming constants $\left\{\alpha_{n}\right\}_{n \in \mathbb{N}_{0}}$ can be constructed from the two sequences of eigenvalues, $\Gamma_{3}:=\left\{\lambda_{n}, \tilde{\lambda}_{n}\right\}_{n \in \mathbb{N}_{0}}$.

The uniqueness problem of determining the potential $q$ in terms of one of the above-mentioned three sets of spectral data, $\Gamma_{j}(j=1,2,3)$, was first investigated by Borg [4, 5], Levitan [25, 26, and Marchenko [29, 30. Further investigation has been carried out by Hald [17, 18, Hochstadt [19], Trubowitz and his co-authors in a series of papers [8, 21, 22, 32, and others. A comprehensive review for the inverse problem in these cases is presented by McLaughlin 31 .

The present paper will mainly investigate the uniqueness problem of the determination of the potential $q$ under the circumstances where only partial information of $q$, of the eigenvalues $\left\{\lambda_{n}\right\}_{n \in \mathbb{N}_{0}}$, and of the norming constants $\left\{\kappa_{n}\right\}_{n \in \mathbb{N}_{0}}$ is available.

In 1978, Hochstadt and Lieberman 20 proved a remarkable result which says that mixed given data (e.g., given one spectrum and $q$ known on $[0,1 / 2])$ can determine $q$ uniquely on $[0,1]$. More precisely, if $q_{1}=q_{2}$ on $[0,1 / 2]$ and if $\sigma\left(L_{1}\left(q_{1} ; h_{0}, h_{1}\right)\right)$ $=\sigma\left(L_{2}\left(q_{2} ; h_{0}, h_{1}\right)\right)$, then $q_{1}=q_{2}$ on $[0,1]$. Later, refinements by Hald [17] and Suzuki [34] of Hochstadt and Lieberman's theorem showed that the boundary conditions at $x=1$ for $L_{1}$ and $L_{2}$ need not be assumed a priori to be the same and that if $q$ is continuous, then one only needs $\lambda_{1, n}=\lambda_{2, m(n)}$ for all values of $n$ but one, that is, one eigenvalue can be missing. However, this is no longer true if the boundary condition at $x=1$ is different and $q$ is discontinuous for all values of $n$ but one (see [16]). The same boundary condition for $L_{1}$ and $L_{2}$ at $x=0$, however, is crucial for Hochstadt and Lieberman's theorem to hold (see [33]).

Several important generalizations of Hochstadt and Lieberman's theorem are given by Gesztesy and Simon [16] who consider the case where the $L^{1}[0,1]$ potential $q$ is known on a larger interval $[0, a]$ with $a \in[1 / 2,1)$ and assume that the set of 
common eigenvalues of $L_{1}$ and $L_{2}$ is sufficiently large. Another result in [16 is to assume that the potential $q$ belongs to $C^{2 k}$ for some $k \in \mathbf{N}_{0}$ near $1 / 2$ so that $C^{2 k}$ smoothness can replace the knowledge of some $(k+1)$ eigenvalues, that is, $(k+1)$ eigenvalues can be missing. In [10, del Rio, Gesztesy and Simon further study the case where $a$ can be any number in the interval $(0,1)$. In other words, they consider the problem of uniquely determining the potential $q$ if $q$ is only known on part of the interval $(0,1)$ (which may be a subinterval of $\left(0, \frac{1}{2}\right)$ ). In such a case, since less information is given on $q$, to uniquely determine the potential $q$ on the entire interval $[0,1]$, one naturally needs more information on the spectra $\sigma\left(L\left(q, h_{0}, h_{1}\right)\right)$ for several different values of $h_{0}$. For instance, $q$ can be completely determined if either of the following two conditions is satisfied [10]:

(a) given the spectrum $\sigma\left(L\left(q, h_{0}, h_{1}\right)\right)$, half of the spectrum $\sigma\left(L\left(q, h_{0}^{\prime}, h_{1}\right)\right)$ for $h_{0} \neq h_{0}^{\prime}$, and $q$ on $[0,1 / 4]$,

(b) given two-thirds of the spectra $\left(q, h_{0}, h_{1}\right)$ for three different values of $h_{0}$.

These results have been generalized and improved in a variety of ways; see [2, 3, 23, 24.

The purpose of this paper is twofold. First we provide some new results, analogous to the theorems of Gesztesy and Simon [15], [16, on the unique determination problem of the potential $q$ and $h_{1}$ for the Sturm-Liouville problem (problems (1.1)-(1.3)) with only partial information available on $q$ and partial spectral data consisting of one full spectrum and a subsequence of norming constants, or a subset of the pairs of eigenvalues and the corresponding norming constants. Furthermore, if the potential $q$ is $C^{2 k-1}$-smooth near the endpoint $x=0$, then the potential $q$ and $h_{1}$ can be determined uniquely from the values of $q^{(j)}(0)$, for $j=0,1, \ldots, 2 k-1$, one full spectrum and the norming constants except for $(k+1)$, that is, $(k+1)$ norming constants can be missing. In particular, if $q \in L^{1}[0,1]$, then one norming constant can be missing.

The above results enlighten us to realize that, for the question of uniqueness for the Sturm-Liouville problem, norming constants play an equal role as eigenvalues. In other words, the number of norming constants but one is, in a sense, equivalent to the number of eigenvalues, which has a connection with the interesting open question raised by Gesztesy and Simon [16, p. 2775]: Can one replace information on the missing eigenvalue by knowledge of the boundary condition $h_{1}$ ? (This question was recently solved in the affirmative [35. in the case of $q \in L^{1}[0,1]$.)

It is therefore the second purpose of this paper to show that if the boundary conditions at the endpoints $x=0$ and 1 are fixed and if $q$ is $C^{2 k-1}$ near $x=0$, then the values of $q$ on the entire interval $[0,1]$ can be uniquely determined by the values of $q^{(j)}(0)$ for $j=0,1, \ldots, 2 k-1$, a set of eigenvalues except for one, and a set of the norming constants except for $(k+1)$, that is, one eigenvalue and $(k+1)$ norming constants can be missing. In particular, in the case of $q \in L^{1}[0,1]$, the boundary condition $h_{1}$ can replace the knowledge of one eigenvalue. This shows that the answer to the above-mentioned open question of Gesztesy and Simon is affirmative in more general spectral data.

The main results presented in this paper are based on the uniqueness theorem of the Weyl $m$-function developed by Marchenko [29, 30. This approach has been employed skillfully by del Rio, Gesztesy and Simon in a series of papers $10,13,14,15,16$, to deal with inverse problems. The key technique relies on 
the asymptotic expansion of an $m$-function. However, since the asymptotic expansion of an $m$-function is independent of boundary conditions, the technique of the Weyl $m$-functions fails to treat the case where boundary conditions are fixed. In Section 5 we shall provide an alternative technique which is similar to a method of Hochstadt [19] and Hald [18.

The paper is organized as follows. In the next section, we recall a uniqueness theorem due to Marchenko 29]. In Section 3, we discuss the case where one full spectrum and a subset of norming constants are known. In Section 4, we treat the case where a subset of pairs of the eigenvalues and of the norming constants are given. In Section 5, we deal with the case where the boundary conditions at the endpoints are the same. Finally, Section 6 is devoted to the case of the Dirichlet boundary condition.

We adopt the notation:

- $\mathbb{R}$ : the set of real numbers;

- $\mathbb{N}$ : the set of positive integers;

- $\mathbb{N}_{0}:\{0\} \cup \mathbb{N}$, i.e., the set of nonnegative integers;

- $\mathbb{C}$ : the complex plane;

- $\mathbb{C}_{+}$: the open complex upper half-plane.

\section{Preliminaries}

Throughout this paper, by the statement " $q$ on $[0, a]$, eigenvalues $\lambda_{n}$, and norming constants $\kappa_{n}$ determine uniquely $q$ and $h_{1}$ " we mean that there are no two distinct potentials $q_{1}$ and $q_{2}$ on $[0,1]$ with the two properties: (i) $q_{1}=q_{2}$ a.e. on $[0, a]$, and (ii) $\lambda_{n}$ and $\kappa_{n}$ are common eigenvalues and norming constants for $q_{1}$ and $q_{2}$. Unless explicitly stated otherwise, $h_{0}$ will be fixed and all potentials $q, q_{1}$, and $q_{2}$ will be real-valued and in $L^{1}[0,1]$ for the rest of this paper.

Moreover, to avoid too many case distinctions in the proofs below, we shall assume $h_{0}, h_{1} \in \mathbb{R}$ in (1.1)-(1.3) throughout the main body of this paper. In particular, for $h_{0}, h_{1} \in \mathbb{R}$ we index the eigenvalues $\lambda_{n}$ of the operator $L$ by $n \in \mathbb{N}_{0}$. The case of Dirichlet boundary conditions, where $h_{0}=\infty$ and/or $h_{1}=\infty$, will be dealt with in Section 6.

For a real-valued potential $q \in L^{1}[0,1]$, consider the initial-value problem

$$
-u^{\prime \prime}+q u=z u
$$

on $[0,1]$ with initial conditions

$$
\begin{array}{ll}
u_{-}(0)=1, & u_{-}^{\prime}(0)=h_{0}, \\
u_{+}(1)=1, & u_{+}^{\prime}(1)=h_{1} .
\end{array}
$$

Let $u_{-}:=u_{-}(x, z)$ and $u_{+}:=u_{+}(x, z)$ be the solutions of problems (2.1)-(2.2) and problems (2.1) and (2.3), respectively. If $z=\lambda_{j} \in \sigma(L)$, where the operator $L$ is defined by problems (1.1)-(1.3), then both $u_{-}\left(x, \lambda_{j}\right)=: u_{-, j}$ and $u_{+}\left(x, \lambda_{j}\right)=: u_{+, j}$ are eigenfunctions, corresponding to the eigenvalue $\lambda_{j}$, of the operator $L$, and there holds the relation

$$
u_{+, j}=\kappa_{j} u_{-, j},
$$

where $\kappa_{j}=u_{+, j}(0)=u_{-, j}^{-1}(1)$ is the norming constant (or ratio) corresponding to the eigenvalue $\lambda_{j}$; hence $\kappa_{j} \neq 0, \infty$. 
We next formulate the main uniqueness theorem in the literature, due to Marchenko [29]. For the solution $u_{+}(x, z)$ of $(2.1)$, the Weyl $m_{+}$-function is defined by

$$
m_{+}(a, z)=\frac{u_{+}^{\prime}(a, z)}{u_{+}(a, z)}
$$

for any $a \in[0,1)$. Similarly, the Weyl $m_{-}$-function is defined by

$$
m_{-}(b, z)=-\frac{u_{-}^{\prime}(b, z)}{u_{-}(b, z)}
$$

for any $b \in(0,1]$. The differing signs in $(2.5)$ and (2.6) are picked so that both $m_{+}$ and $m_{-}$are Herglotz functions, that is, $m_{ \pm}: \mathbb{C}_{+} \rightarrow \mathbb{C}_{+}$are analytic.

Marchenko's 29] fundamental uniqueness theorem of inverse spectral theory then reads as follows:

Theorem 2.1. $m_{+}(a, z)$ (resp., $\left.m_{-}(b, z)\right)$ uniquely determines $h_{1}$ (resp., $\left.h_{0}\right)$ as well as $q$ (a.e.) on $[a, 1]$ (resp., $[0, b])$.

Consider a problem with boundary condition (1.3) at $x=1$. We need to know the high-energy asymptotic behavior of the $m_{+}$-function with $x \in[0,1)$. It is known [1, 11] that, under the general hypothesis $q \in L^{1}[0,1]$,

$$
m_{+}(x, z)=i \sqrt{z}+o(1), \quad \frac{1}{m_{+}(x, z)}=\frac{-i}{\sqrt{z}}+o\left(\frac{1}{z}\right)
$$

uniformly in $x \in[0,1-\delta]$ for $\delta>0$ as $|z| \rightarrow \infty$ in any sector $\varepsilon<\operatorname{Arg}(z)<\pi-\varepsilon$ for $\varepsilon>0$, where $\sqrt{z}$ is the square root branch with $\operatorname{Im}(\sqrt{z}) \geq 0$. It is also known [9] that if $q$ is $C^{n}$ near $x_{0} \in[0,1)$, for some $n \in \mathbb{N}_{0}$, then $m_{+}\left(x_{0}, z\right)$ and $m_{+}\left(x_{0}, z\right)^{-1}$ have asymptotic expansions of the form

$$
\begin{aligned}
& m_{+}\left(x_{0}, z\right)=i \sqrt{z}+\sum_{l=1}^{n+1} c_{l}\left(x_{0}\right) \frac{1}{z^{(l+1) / 2}}+o\left(\frac{1}{z^{(n+1) / 2}}\right), \\
& \frac{1}{m_{+}\left(x_{0}, z\right)}=\frac{-i}{\sqrt{z}}+\sum_{l=1}^{n+1} d_{l}\left(x_{0}\right) \frac{1}{z^{(l+2) / 2}}+o\left(\frac{1}{z^{(n+3) / 2}}\right)
\end{aligned}
$$

as $|z| \rightarrow \infty$ in any sector $\varepsilon<\operatorname{Arg}(z)<\pi-\varepsilon$ for $\varepsilon>0$. Here $c_{l}\left(x_{0}\right)$ and $d_{l}\left(x_{0}\right)$ are the universal functions of $q\left(x_{0}\right), q^{\prime}\left(x_{0}\right), \ldots, q^{(l-2)}\left(x_{0}\right)$ and can be computed recursively as follows. Consider the Riccati-type equations for $m_{+}(x, z)$ and $m_{+}(x, z)^{-1}$,

$$
\begin{aligned}
m_{+}^{\prime}(x, z) & =-m_{+}(x, z)^{2} q(x)-z \\
\left(m_{+}(x, z)^{-1}\right)^{\prime} & =-m_{+}(x, z)^{-2}(q(x)-z)+1 .
\end{aligned}
$$


Inserting the asymptotic expansions (2.8) and (2.9) into (2.10) and (2.11) yields the recursive relations

$$
\begin{aligned}
& c_{0}(x)=1, \quad c_{1}(x)=0, \quad c_{2}(x)=-\frac{1}{2} q(x), \\
& c_{j}(x)=\frac{i}{2} c_{j-1}^{\prime}(x)-\frac{1}{2} \sum_{l=1}^{j-1} c_{l}(x) c_{j-l}(x), \quad j \geq 3,
\end{aligned}
$$

$$
\begin{aligned}
& d_{0}(x)=1, \quad d_{1}(x)=0, \quad d_{2}(x)=-\frac{1}{2} q(x), \\
& d_{j}(x)=\frac{i}{2} d_{j-1}^{\prime}(x)-\frac{1}{2} q(x) \sum_{l=1}^{j-1} d_{l}(x) d_{j-l-2}(x)-\frac{1}{2} \sum_{l=1}^{j-1} d_{l}(x) d_{j-l}(x), \quad j \geq 3 .
\end{aligned}
$$

Finally, we introduce the Green's formula (see [36]) associating with (2.1) for a real-valued $q \in L^{1}[0,1]$. Let

$$
D_{\max }:=\left\{y \in L^{2}[0,1]: y, y^{\prime} \in A C[0,1],-y^{\prime \prime}+q y \in L^{2}[0,1]\right\},
$$

where $A C[0,1]$ is the space of absolutely continuous functions on $[0,1]$. Then the Green's formula is stated as follows:

$$
\int_{0}^{1}(y L(z)-z L(y)) d t=[y, z](1)-[y, z](0),
$$

where $y, z \in D_{\max }$ and $[y, z](t):=y(t) z^{\prime}(t)-y^{\prime}(t) z(t)$ for $t \in[0,1]$. It is obvious that if both $u_{1}$ and $u_{2}$ are solutions of $(2.1)$, then $\left[u_{1}, u_{2}\right](x)\left(=\left[u_{1}, u_{2}\right](0)\right)$ is a constant for all $x \in[0,1]$. Here, we mention the precise definition of the Sturm-Liouville operator $L$ in $L^{2}[0,1]: L y=-y^{\prime \prime}+q y$, where

$$
y \in D(L):=\left\{y \in D_{\max }: y \text { satisfies the boundary conditions (1.2)-(1.3) }\right\} \text {. }
$$

\section{The CASE OF ONE FULL SPECTRUM}

In this section, we treat the case where the potential $q$ and the coefficient $h_{1}$ can be uniquely determined if we are given a set of eigenvalues $\lambda_{j}$ and a subset of the corresponding norming constants $\kappa_{j}$. Our results show that if the potential $q$ satisfies a local smoothness condition at $x=0$, then we require less knowledge on the norming constants $\kappa_{j}$. We shall first formulate some asymptotic expansions of solutions of (2.1), which will be used later on to establish our principal results of the section.

Let $q$ be given on $[0, a]$ with some $a \in[0,1)$. Let $q_{1}$ and $q_{2}$ be two candidates for $q$ extended to all of $[0,1]$. Let $u_{1,+}(x, z)$ and $u_{2,+}(x, z)$ be solutions of $(2.1)$ corresponding to $q_{1}$ and $q_{2}$, respectively, where $u_{j,+}(x, z)$ satisfies the initial conditions

$$
u_{j,+}(1, z)=1, \quad u_{j,+}^{\prime}(1, z)=h_{j}, \quad j=1,2,
$$


with $h_{1}, h_{2} \in \mathbb{R}$. It is well known [32, p. 10; [12, [28] that for each $x \in[0,1]$, $u_{j,+}(x, z)$ and $u_{j,+}^{\prime}(x, z)$ are entire functions of $z$ and satisfy the following asymptotic expansions:

$$
\begin{aligned}
& u_{j,+}(x, z)=\cos (\sqrt{z}(1-x))+O\left(e^{\operatorname{Im}(\sqrt{z})(1-x)} \sqrt{z}\right), \\
& u_{j,+}^{\prime}(x, z)=\sqrt{z} \sin (\sqrt{z}(1-x))+O\left(e^{\operatorname{Im}(\sqrt{z})(1-x)}\right)
\end{aligned}
$$

as $|z| \rightarrow \infty$ for all $x \in[0,1]$. The zeros of $u_{j,+}(x, \cdot)$ and of $u_{j,+}^{\prime}(x, \cdot)$ are all real for any $x \in[0,1]$, and they are uniformly bounded below. In particular, we infer that if $z=i y$ with $y \in \mathbb{R}$, then (3.2) and (3.3) imply

$$
\begin{aligned}
\left|u_{j,+}(a, i y)\right| & =\frac{1}{2} e^{\operatorname{Im}(\sqrt{i})(1-a)|y|^{1 / 2}}(1+o(1)) \\
\left|u_{j,+}^{\prime}(a, i y)\right| & =\frac{1}{2}|y|^{1 / 2} e^{\operatorname{Im}(\sqrt{i})(1-a)|y|^{1 / 2}}(1+o(1))
\end{aligned}
$$

as $y \rightarrow \infty$. For $j=1,2$, let

$$
\omega_{j}(z)=u_{j,+}^{\prime}(0, z)-h_{0} u_{j,+}(0, z)
$$

which is called the characteristic function of the operator $L_{j}$. Then $\sigma\left(L_{j}\right)=$ $\left\{\lambda_{j, n}\right\}_{n=0}^{\infty}$ are precisely the zeros of $\omega_{j}(z)$. By (3.4) and (3.5) we also infer that

$$
\left|\omega_{j}(i y)\right|=\frac{1}{2}|y|^{1 / 2} e^{\operatorname{Im}(\sqrt{i})|y|^{1 / 2}}(1+o(1)) \quad \text { as } y \rightarrow \infty
$$

and, for sufficiently large $n$,

$$
\inf _{\theta \in[0,2 \pi]} \mid \omega_{j}\left(\left(\left(\pi\left(n+\frac{1}{2}\right)\right)^{2} e^{i \theta}\right) \mid \geq \pi n+O(1) .\right.
$$

Since the zeros of $u_{j,+}(a, \cdot)$ and of $u_{j,+}^{\prime}(a, \cdot)$ are all real and uniformly bounded below, by adding (if necessary) a sufficiently large constant to $q_{1}$ and $q_{2}$, we may assume that all zeros of $u_{j,+}(a, \cdot), u_{j,+}^{\prime}(a, \cdot)$ and $\omega_{j}(\cdot)$ are in $[1, \infty)$. In this case, all these six functions are of $m$-type (see [16, p. 2781]). Therefore, $u_{j,+}(a, \cdot), u_{j,+}^{\prime}(a, \cdot)$ and $\omega_{j}(\cdot)$ are bounded by $C_{1} \exp \left(C_{2}|z|^{1 / 2}\right)$ for some constant $C_{1}, C_{2}>0$ and are of the form (see [16])

$$
c \prod_{n=0}^{\infty}\left(1-\frac{z}{x_{n}}\right)
$$

for suitable $\left\{x_{n}\right\}_{n=0}^{\infty} \subset[1, \infty)$.

For any $\alpha=\left\{\alpha_{j}\right\}_{j \in \mathbb{N}_{0}} \subset \mathbb{C}$, set

$$
n_{\alpha}(t)=\#\left\{j \in \mathbb{N}_{0}:\left|\alpha_{j}\right| \leq t\right\} \quad \text { for each } t \geq 0 .
$$

Here is one of our main results of this section.

Theorem 3.1. Let $\Lambda_{0}=\left\{j_{1}, j_{2}, \ldots, j_{k+1}\right\} \subset \mathbb{N}_{0}$. Suppose that $q_{1}, q_{2} \in C^{2 k-1}[0, \varepsilon)$ for some $k \in \mathbb{N}_{0}$ and for some $\varepsilon>0$. Assume that

$$
\begin{gathered}
q_{1}^{(j)}(0)=q_{2}^{(j)}(0), \quad j=0,1, \ldots, 2 k-1, \\
\sigma\left(L_{1}\right)=\sigma\left(L_{2}\right) \quad \text { and } \quad \kappa_{1, j}=\kappa_{2, j}, j \in \mathbb{N}_{0} \backslash \Lambda_{0} .
\end{gathered}
$$

Then $h_{1}=h_{2}$ and $q_{1}=q_{2}$ a.e. on $[0,1]$. 
Remark 3.2. Under the assumption that $\sigma\left(L_{1}\right)=\sigma\left(L_{2}\right)$, it is known [12, p. 8] that $\omega_{1}(z)=\omega_{2}(z)$; see also (3.13) below. By the fact that $\kappa_{l, j} \alpha_{l, j}=\omega_{l}^{\prime}\left(\lambda_{j}\right)$ for $l=1,2$ and $j \in \mathbb{N}_{0}$, we easily find that the result of Theorem 3.1 remains valid if the condition $\kappa_{1, j}=\kappa_{2, j}$ is replaced with the condition $\alpha_{1, j}=\alpha_{2, j}$, for all $j \in \mathbb{N}_{0} \backslash \Lambda_{0}$.

Comparing the result of Theorem 3.1 with that of [16, Theorem 1.2], one may see that the $(k+1)$ missed eigenvalues (as $\left.q \in C^{2 k}[0, \varepsilon)\right)$ replaced by the $(k+1)$ missed norming constants (as $q \in C^{2 k-1}[0, \varepsilon)$ ) can also uniquely determine $q$ and $h_{1}$. In particular, if $q \in L^{1}[0,1]$, by Theorem 3.1, we have

Corollary 3.3. Let $q \in L^{1}[0,1]$. Then one full spectral data $\left\{\lambda_{j}, \kappa_{j}\right\}_{j \in \mathbb{N}_{0}}$ except for one norming constant determines the potential $q$ and $h_{1}$ uniquely.

Proof of Theorem 3.1. Let $\sigma\left(L_{1}\right)=\sigma\left(L_{2}\right)=:\left\{\lambda_{j}\right\}_{j \in \mathbb{N}_{0}}$ be the set of all the common eigenvalues of $L_{1}$ and $L_{2}$. Then $\left\{\lambda_{j}\right\}_{j \in \mathbb{N}_{0}}$ are precisely the zeros of $\omega_{l}(z)$ for $l=1,2$, and by [12, p. 8] we have

$$
\omega_{1}(z)=\left(\lambda_{0}-z\right) \prod_{j=1}^{\infty} \frac{\lambda_{j}-z}{j^{2}}=\omega_{2}(z) .
$$

Let $v_{2,+}(x, z)$ be another solution of the equation $-u^{\prime \prime}+q_{2} u=z u$ subject to the initial condition

$$
v_{2,+}(1, z)=1, \quad v_{2,+}^{\prime}(1, z)=h
$$

with $h \in \mathbb{R}$ and $h \neq h_{1}, h_{2}$. It is easy to verify that $v_{2,+}$ satisfies the boundary condition at $x=1$,

$$
v_{2,+}^{\prime}(1, z)-h v_{2,+}(1, z)=0
$$

Let

$$
\widetilde{\omega}(z):=v_{2,+}^{\prime}(0, z)-h_{0} v_{2,+}(0, z) .
$$

Consider the operator $L\left(q_{2}, h_{0}, h\right)$ in $L^{2}[0,1]$ with boundary conditions (1.2) and (1.3), where $h_{1}$ is replaced by $h$. Then $L\left(q_{2}, h_{0}, h\right)$ is self-adjoint and its spectrum consists of eigenvalues, denoted by $\left\{\mu_{j}\right\}_{j \in \mathbb{N}_{0}}$, which are real, simple and bounded from below. It is also known [36, Ch. 4] that the spectrum of $L\left(q_{2}, h_{0}, h\right)$ and $L\left(q_{2}, h_{0}, h_{2}\right)$ are interlaced and disjoint, since $h \neq h_{2}$.

Now we consider the function $F(z)$ defined by

$$
F(z)=\left(u_{1,+}(0, z)-u_{2,+}(0, z)\right) \widetilde{\omega}(z) .
$$

It follows from (3.15) and (3.14) that $F\left(\mu_{j}\right)=0$ for all $j \in \mathbb{N}_{0}$. Furthermore, it is known (see (2.4)) that $u_{l,+}\left(0, \lambda_{j}\right)=\kappa_{l, j}$ for $l=1,2$ and thus, $F\left(\lambda_{j}\right)=0$ if $\lambda_{j} \in \mathbb{N}_{0} \backslash \Lambda_{0}$. Noting that $\omega_{1}(z)=\omega_{2}(z)$ and by (3.15) and (2.5), we get

$$
\begin{aligned}
F(z)= & {\left[\left(u_{1,+}(0, z)\left(v_{2,+}^{\prime}(0, z)-h_{0} v_{2,+}(0, z)\right)-v_{2,+}(0, z) \omega_{1}(z)\right)\right] } \\
& -\left[u_{2,+}(0, z)\left(v_{2,+}^{\prime}(0, z)-h_{0} v_{2,+}(0, z)\right)-v_{2,+}(0, z) \omega_{2}(z)\right] \\
= & {\left[u_{1,+}, v_{2,+}\right](0, z)-\left[u_{2,+}, v_{2,+}\right](0, z) } \\
= & u_{1,+}^{\prime}(0, z) v_{2,+}^{\prime}(0, z)\left(m_{+, v_{2,+}}(0, z)^{-1}-m_{+, u_{1,+}}(0, z)^{-1}\right) \\
& -u_{2,+}^{\prime}(0, z) v_{2,+}^{\prime}(0, z)\left(m_{+, v_{2,+}}(0, z)^{-1}-m_{+, u_{2,+}}(0, z)^{-1}\right),
\end{aligned}
$$


where $m_{+, v_{2,+}}(0, z)=v_{2,+}^{\prime}(0, z) / v_{2,+}(0, z)$ is the Weyl $m$-function associated with the solution $v_{2,+}(\operatorname{see}(2.5))$. Define

$$
H(z)=\frac{F(z)}{G(z)} \prod_{l=1}^{k+1}\left(z-\lambda_{j_{l}}\right)
$$

where $j_{l} \in \Lambda_{0}$ and $G(z)=\omega_{2}(z) \widetilde{\omega}(z)$. By the above discussions, the cross ratio $F(z) \prod_{l=1}^{k+1}\left(z-\lambda_{j_{l}}\right)$ vanishes at each point where $G$ vanishes. Also, $G$ necessarily has simple zeros, since $L\left(q_{2}, h_{0}, h_{2}\right)$ and $L\left(q_{2}, h_{0}, h\right)$ have simple spectrum, respectively, and their spectrum are interlaced and disjoint. Thus, $H$ is an entire function. In addition, from (3.8) and the fact that the functions $u_{j,+}(0, z)$ and $v_{2,+}(0, z)$ both are of $m$-type, we conclude that $H(z)$ satisfies

$$
|H(z)| \leq C_{1} e^{C_{2}|z|^{1 / 2}}
$$

As a matter of fact, it follows from (3.8) that the above inequality holds whenever $|z|=(\pi(n+1 / 2))^{2}$ for $n$ sufficiently large; it then extends to all $z$ by the maximum modulus principle. Furthermore, since $q_{1}^{(j)}(0)=q_{2}^{(j)}(0)$ for $j=0,1, \ldots, 2 k-1$, by (2.9) we infer that

$$
\begin{aligned}
& \left|m_{+, v_{2,+}}(0, i y)^{-1}-m_{+, u_{1,+}}(0, i y)^{-1}\right|=o\left(|y|^{-(k+1)}\right), \\
& \left|m_{+, v_{2,+}}(0, i y)^{-1}-m_{+, u_{2,+}}(0, i y)^{-1}\right|=o\left(|y|^{-(k+1)}\right) .
\end{aligned}
$$

This together with (3.5), (3.18) and (3.7) shows that

$$
\begin{aligned}
|H(i y)| \leq & \left|\frac{\left(u_{1,+}^{\prime} v_{2,+}^{\prime}\right)(0, i y)\left(m_{+, v_{2,+}}(0, i y)^{-1}-m_{+, u_{1,+}}(0, i y)^{-1}\right)}{G(i y)} \prod_{l=1}^{k+1}\left(i y-\lambda_{j_{l}}\right)\right| \\
& +\left|\frac{\left(u_{2,+}^{\prime} v_{2,+}^{\prime}\right)(0, i y)\left(m_{+, v_{2,+}}(0, i y)^{-1}-m_{+, u_{2,+}}(0, i y)^{-1}\right)}{G(i y)} \prod_{l=1}^{k+1}\left(i y-\lambda_{j_{l}}\right)\right| \\
= & \frac{|y| e^{\operatorname{Im}(\sqrt{i}) 2|y|^{1 / 2}}(1+o(1)) o\left(|y|^{-(k+1)}\right)}{|y| e^{\operatorname{Im}(\sqrt{i}) 2|y|^{1 / 2}}(1+o(1))} O\left(|y|^{(k+1)}\right) \\
(3.21)= & o(1) \quad(y(\operatorname{real}) \rightarrow \infty) .
\end{aligned}
$$

It turns out that $|H(i y)| \rightarrow 0$ as $y$ (real) $\rightarrow \infty$. By [16. Proposition B.6] and (3.21), we obtain $H \equiv 0$. We can multiply $H$ by $(\omega \widetilde{\omega})(z) / \prod_{l=1}^{k+1}\left(z-\lambda_{j_{l}}\right)$, which has isolated zeros and poles, to conclude that $u_{1,+}(0, z)=u_{2,+}(0, z)$ for all $z \in \mathbb{C}$. This together with (3.13) and (3.6) yields $u_{1,+}^{\prime}(0, z)=u_{2,+}^{\prime}(0, z)$, and therefore $m_{+, u_{2,+}}(z)=m_{+, u_{1,+}}(z)$. By Theorem 2.1, $q_{1}=q_{2}$ a.e. on $[0,1]$, as well as $h_{1}=h_{2}$. The proof is therefore complete.

The following theorem treats the case where partial information is given on the set of the norming constants when $q$ is known a priori on $[0, a]$ with $a \in(0,1 / 2)$.

Theorem 3.4. Let $a \in(0,1 / 2)$ and $q_{1}, q_{2} \in C^{n}(a-\varepsilon, a+\varepsilon)$ for some $n \in \mathbb{N}_{0}$ and for some $\varepsilon>0$. Consider a subset $S \subseteq \sigma\left(L_{2}\right)$ such that $\kappa_{1, j}=\kappa_{2, j}$ for $\lambda_{j} \in S$. Assume that $q_{1}=q_{2}$ a.e. on $[0, a], \sigma\left(L_{1}\right)=\sigma\left(L_{2}\right)$ and

$$
n_{S}(t) \geq(1-2 a) n_{\sigma\left(L_{2}\right)}(t)+a-\frac{n+3}{2}
$$

for all sufficiently large $t \in \mathbb{R}$. Then $h_{1}=h_{2}$ and $q_{1}=q_{2}$ a.e. on $[0,1]$. 
Remark 3.5. If both $q_{1}$ and $q_{2}$ are assumed to be in $L^{1}[0,1]$, then, instead of condition (3.22), we only need the following condition:

$$
n_{S}(t) \geq(1-2 a) n_{\sigma\left(L_{1}\right)}(t)+a-1 .
$$

Remark 3.6. As a typical example, knowing one full spectrum and a half set of the norming constants and also knowing $q$ on $\left[0, \frac{1}{4}\right]$ uniquely determines $q$ on all of $[0,1]$ and $h_{1}$. To the best of our knowledge, Theorem 3.4 solves a new type of inverse spectral problem involving fractions of the set of norming constants as in (3.22), parallel to Gesztesy and Simon's result [16, Theorem 1.3] on inverse spectral problems involving fractions of the set of eigenvalues.

Proof of Theorem 3.4. Consider the function $F$ defined by

$$
F(z)=\left(u_{1,+}(0, z)-u_{2,+}(0, z)\right) \widetilde{\omega}(z),
$$

where $\widetilde{\omega}(z)$ is defined by (3.15). Then by the hypothesis that $\sigma\left(L_{1}\right)=\sigma\left(L_{2}\right)=$ : $\left\{\lambda_{j}\right\}_{j \in \mathbb{N}_{0}}$ and the argument of the proof of Theorem 3.1, we get

$$
F\left(\lambda_{j}\right)=0 \text { if } \lambda_{j} \in S, \quad F\left(\mu_{j}\right)=0 \text { if } \mu_{j} \in \sigma\left(L\left(q_{2}, h_{0}, h\right)\right) .
$$

Let $a \in[0,1 / 2)$. Since $q_{1}=q_{2}$ on $[0, a]$ we infer by the Green's formula (2.14) that

$$
\begin{aligned}
{\left[u_{2,+}, v_{2,+}\right](0, z) } & =\left[u_{2,+}, v_{2,+}\right](a, z), \\
{\left[u_{1,+}, v_{2,+}\right](0, z) } & =\left[u_{1,+}, v_{2,+}\right](a, z)-\int_{0}^{a}\left(q_{1}-q_{2}\right)(t)\left(u_{1,+} v_{2,+}\right)(t, z) d t \\
& =\left[u_{1,+}, v_{2,+}\right](a, z) .
\end{aligned}
$$

This, combined with (3.17) and (2.5), shows that

$$
\begin{aligned}
F(z)= & {\left[u_{1,+}, v_{2,+}\right](a, z)-\left[u_{2,+}, v_{2,+}\right](a, z) } \\
= & u_{1,+}^{\prime}(a, z) v_{2,+}^{\prime}(a, z)\left(m_{+, v_{2,+}}(a, z)^{-1}-m_{+, u_{1,+}}(a, z)^{-1}\right) \\
& -u_{2,+}^{\prime}(a, z) v_{2,+}^{\prime}(a, z)\left(m_{+, v_{2,+}}(a, z)^{-1}-m_{+, u_{2,+}}(a, z)^{-1}\right) .
\end{aligned}
$$

Define

$$
G_{S}(z)=\prod_{\lambda_{j} \in S}\left(1-\frac{z}{\lambda_{j}}\right), \quad G_{\sigma\left(L_{2}\right)}(z)=\prod_{\lambda_{j} \in \sigma\left(L_{2}\right)}\left(1-\frac{z}{\lambda_{j}}\right),
$$

where $S \subset \sigma\left(L_{2}\right)$. By the hypothesis (3.22) on $S$ we have, by integration by parts,

$$
\begin{aligned}
\ln \left|G_{S}(i y)\right| & =\sum_{\lambda_{j} \in S} \frac{1}{2} \ln \left(1+\frac{y^{2}}{\lambda_{j}^{2}}\right) \\
& =\frac{1}{2} \int_{0}^{\infty} \ln \left(1+\frac{y^{2}}{t^{2}}\right) d n_{S}(t) \\
& =\int_{0}^{\infty} \frac{y^{2}}{t^{3}+t y^{2}} n_{S}(t) d t \quad\left(\text { as } n_{S}(0)=0\right) \\
& =\int_{1}^{\infty} \frac{y^{2}}{t^{3}+t y^{2}} n_{S}(t) d t \quad\left(n_{S}(t)=0 \text { if } t \in[0,1)\right) .
\end{aligned}
$$

Furthermore, by hypothesis (3.22) of the theorem, there are constants $t_{0} \geq 1$ and $C \geq 0$ such that

$$
n_{S}(t) \geq \begin{cases}(1-2 a) n_{\sigma\left(L_{2}\right)}(t)+a-(n+3) / 2, & \text { if } t>t_{0}, \\ (1-2 a) n_{\sigma\left(L_{2}\right)}(t)-C, & \text { if } t \leq t_{0} .\end{cases}
$$


Hence, by (3.27) and (3.22), and noting the relation

$$
\frac{y^{2}}{t^{3}+t y^{2}}=-\frac{d}{d t}\left(\frac{1}{2} \ln \left(1+\frac{y^{2}}{t^{2}}\right)\right)
$$

we deduce that

$$
\begin{aligned}
\ln \left|G_{S}(i y)\right|= & \int_{1}^{t_{0}} \frac{y^{2}}{t^{3}+t y^{2}} n_{S}(t) d t+\int_{t_{0}}^{\infty} \frac{y^{2}}{t^{3}+t y^{2}} n_{S}(t) d t \\
\geq & (1-2 a) \int_{1}^{\infty} \frac{y^{2}}{t^{3}+t y^{2}} n_{\sigma\left(L_{2}\right)}(t) d t \\
& +\left(a-\frac{n+3}{2}\right) \int_{1}^{\infty} \frac{y^{2}}{t^{3}+t y^{2}} d t+C_{0} \\
= & (1-2 a) \ln \left|G_{\sigma\left(L_{2}\right)}(i y)\right|+\frac{1}{2}(a-(n+3) / 2) \ln \left(1+y^{2}\right)+C_{0},
\end{aligned}
$$

where $C_{0}=|a-(n+3) / 2|+C \ln \left(t_{0}\right)$.

Because $\sigma\left(L_{2}\right)$ is the full set of the eigenvalues of the self-adjoint operator $L_{2}$ on $[0,1]$, we know that asymptotically

$$
\left|G_{\sigma\left(L_{2}\right)}(i y)\right|=\frac{1}{2}|y|^{1 / 2} e^{\operatorname{Im}(\sqrt{i})|y|^{1 / 2}}(1+o(1)) \quad \text { as } y(\text { real }) \rightarrow \infty .
$$

It thus turns out from (3.28) that there exists a positive constant $C_{1}$ such that

$$
\left|G_{S}(i y)\right| \geq C_{1}|y|^{-(n+2) / 2} e^{\operatorname{Im}(\sqrt{i})(1-2 a)|y|^{1 / 2}}
$$

for $|y|$ sufficiently large. Let

$$
H(z)=\frac{F(z)}{G(z)}
$$

where $G(z)=G_{S}(z) \widetilde{\omega}(z)$. Recall that

$$
\left|u_{j,+}^{\prime}(a, i y)\right|=\frac{1}{2} e^{\operatorname{Im}(\sqrt{i})(1-a)|y|^{1 / 2}}(1+o(1)), \quad j=1,2 .
$$

Note that $v_{2,+}^{\prime}(a, i y)$ has the same asymptotics as $u_{j,+}^{\prime}(a, i y)$. Thus, by (3.25), (3.20) and (3.29), we have

$$
\begin{aligned}
|H(i y)| \leq & \left|\frac{u_{1,+}^{\prime}(a, i y) v_{2,+}^{\prime}(a, i y)\left(m_{+, v_{2,+}}(a, i y)^{-1}-m_{+, u_{1,+}}(a, i y)^{-1}\right)}{G(z)}\right| \\
& +\left|\frac{u_{2,+}^{\prime}(a, i y) v_{2,+}^{\prime}(a, i y)\left(m_{+, v_{2,+}}(a, i y)^{-1}-m_{+, u_{2,+}}(a, i y)^{-1}\right)}{G(z)}\right| \\
\leq & \frac{|y| e^{\operatorname{Im}(\sqrt{i}) 2(1-a)|y|^{1 / 2}}(1+o(1)) o\left(|y|^{-(n+3) / 2}\right)}{|y|^{1 / 2} e^{\operatorname{Im}(\sqrt{i})|y|^{1 / 2}}(1+o(1))|y|^{-(n+2) / 2} e^{\operatorname{Im}(\sqrt{i})(1-2 a)|y|^{1 / 2}}} \\
= & o(1) .
\end{aligned}
$$

This yields $H(z)=0$ and therefore $F(z)=0$ for all $z \in \mathbb{C}$ by the argument of the proof of Theorem 3.1. This together with (3.24) and (3.13) yields $m_{+, u_{2,+}}(z)=$ $m_{+, u_{1,+}}(z)$. By Theorem 2.1, $q_{1}=q_{2}$ a.e. on $[0,1]$ and $h_{1}=h_{2}$. The proof is complete. 
Remark 3.7. By checking the proofs of Theorems 3.1 and 3.4, we easily see that the results of Theorems 3.1 and 3.4 are independent of the boundary condition $h$ at $x=1$ of the solution $v_{2,+}(x, z)$, even if the boundary condition is the Dirichlet boundary condition (i.e., $h=\infty$ ).

\section{PAirs of EIGENVALUES AND NORMING CONSTANTS}

In this section, we study the case where we can use a subset $\left\{\lambda_{j}, \kappa_{j}: j \in \Lambda \subset \mathbb{N}_{0}\right\}$ of the (full) set $\left\{\lambda_{j}, \kappa_{j}: j \in \mathbb{N}_{0}\right\}$ of eigenvalues and the corresponding norming constants to uniquely determine $q$ and $h_{1}$. The method used here is similar to the method used in the last section.

Theorem 4.1. Let $a \in[0,1)$ and $q_{1}, q_{2} \in L^{1}[0,1]$. Consider a subset $S \subseteq \sigma\left(L_{1}\right) \cap$ $\sigma\left(L_{2}\right)$ such that $\kappa_{1, j}=\kappa_{2, j}$ for $\lambda_{j} \in S$. Assume $q_{1}=q_{2}$ a.e. on $[0, a]$ and

$$
n_{S}(t) \geq(1-a) n_{\sigma\left(L_{2}\right)}(t)-\frac{1}{2}(1-a)
$$

for all sufficiently large $t \in \mathbb{R}$. Then $h_{1}=h_{2}$ and $q_{1}=q_{2}$ a.e. on $[0,1]$.

Remark 4.2. Let $a=1 / 2$ and let $K \geq 0$ be any fixed integer. A consequence of Theorem 4.1 is the following. To uniquely determine $q$ on $[0,1]$ and $h_{1}$, we need half data from the set $\left\{\lambda_{j}, \kappa_{j}: 0 \leq j \leq K\right\}$ (the other half can be missing) plus either $\left\{\lambda_{2 j}, \kappa_{2 j}: 2 j \geq K\right\}$ or $\left\{\lambda_{2 j-1}, \kappa_{2 j-1}: 2 j-1 \geq K\right\}$. In particular, we have the following result.

Corollary 4.3. Let $a=1 / 2$. Then we need half of the (full) set $\left\{\lambda_{j}, \kappa_{j} ; j \in \mathbb{N}_{0}\right\}$ of eigenvalues and the corresponding norming constants to uniquely determine $q$ on $[0,1]$ and $h_{1}$. That is, the even (resp., odd) spectral data $\left\{\lambda_{2 j}, \kappa_{2 j}\right\}_{j \geq 0}$ (resp., $\left.\left\{\lambda_{2 j+1}, \kappa_{2 j+1}\right\}_{j \geq 0}\right)$ and $q$ on $[0,1 / 2]$ can uniquely determine $h_{1}$ and $q$ on $[0,1]$.

Proof of Theorem 4.1. Let us consider the function

$$
F_{v}(z):=\left(h_{1}-h_{2}\right) v_{2}(1, z)-\int_{0}^{1}\left(q_{1}-q_{2}\right)(x)\left(u_{1,+} v_{2}\right)(x, z) d x,
$$

where $v_{2}(x, z)$ is a solution of the equation

$$
-u^{\prime \prime}+q_{2} u=z u \quad \text { on }[0,1] .
$$

Then by the Green's formula (2.14) and the initial conditions (3.1) of $u_{1,+}$ and $u_{2,+}$ we have

$$
\begin{aligned}
& F_{v}(z)=\left(h_{1}-h_{2}\right) v_{2}(1, z)-\int_{0}^{1}\left[\left(u_{1,+}^{\prime \prime}+z u_{1,+}\right) v_{2}-\left(v_{2}^{\prime \prime}+z v_{2}\right) u_{1,+}\right](x, z) d x \\
& =\left(h_{1}-h_{2}\right) v_{2}(1, z)+\int_{0}^{1} \frac{d}{d x}\left(\left[u_{1,+}, v_{2}\right](x, z)\right) d x \\
& =\left(u_{1,+}^{\prime}-u_{2,+}^{\prime}\right)(1, z) v_{2}(1, z)+\left[u_{1,+}, v_{2}\right](1, z)-\left[u_{1,+}, v_{2}\right](0, z) \\
& =\left[u_{2,+}, v_{2}\right](1, z)-\left[u_{1,+}, v_{2}\right](0, z) \\
& =\left[u_{1,+}-u_{2,+}, v_{2}\right](0, z) \\
& =\left|\begin{array}{cc}
u_{1,+}(0, z)-u_{2,+}(0, z) & v_{2}(0, z) \\
\omega_{1}(z)-\omega_{2}(z) & v_{2}^{\prime}(0, z)-h_{0} v_{2}(0, z)
\end{array}\right| .
\end{aligned}
$$


Note that $u_{l,+}\left(0, \lambda_{j}\right)=\kappa_{l, j}$ for $l=1,2$. This shows that if $\lambda_{j} \in S$, then $\omega_{1}\left(\lambda_{j}\right)=$ $\omega_{2}\left(\lambda_{j}\right)(=0)$ and $u_{1,+}\left(0, \lambda_{j}\right)=u_{2,+}\left(0, \lambda_{j}\right)$, and therefore $F_{v}\left(\lambda_{j}\right)=0$. Furthermore, since $q_{1}=q_{2}$ on $[0, a]$, it follows from (4.2) and the Green's formula that

$$
\begin{aligned}
F_{v}(z) & =\left(u_{1,+}^{\prime}-u_{2,+}^{\prime}\right)(1, z) v_{2}(1, z)+\int_{a}^{1} \frac{d}{d x}\left(\left[u_{1,+}, v_{2}\right](x, z)\right) d x \\
& =\left[u_{2,+}, v_{2}\right](1, z)-\left[u_{1,+}, v_{2}\right](a, z) \\
& =\left[u_{1,+}-u_{2,+}, v_{2}\right](a, z) .
\end{aligned}
$$

Let $v_{D}(x, z):=v_{2}$ be the solution of (4.3) with the initial conditions $v(a, z)=0$ and $v^{\prime}(a, z)=1$. Then

$$
F_{v_{D}}(z)=\left(u_{1,+}-u_{2,+}\right)(a, z)
$$

and by (3.2) we have

$$
\left|\left(u_{1,+}-u_{2,+}\right)(a, i y)\right|=O\left(|y|^{-1 / 2} e^{\operatorname{Im}(\sqrt{i})(1-a)|y|^{1 / 2}}\right) .
$$

Define

$$
G_{S}(z)=\prod_{\lambda_{j} \in S}\left(1-\frac{z}{\lambda_{j}}\right), \quad H_{D}(z)=\frac{F_{v_{D}}(z)}{G_{S}(z)}
$$

where $S \subset \sigma\left(L_{2}\right)$. By the hypothesis (4.1) on $S$ and the arguments of the proof of Theorem 3.4 (see (3.27) and (3.28)), we have

$$
\left|G_{S}(i y)\right| \geq C_{0} e^{\operatorname{Im}(\sqrt{i})(1-a)|y|^{1 / 2}}
$$

and, in addition, from (4.6),

$$
\left|H_{D}(i y)\right| \leq \frac{O\left(|y|^{-1 / 2} e^{\operatorname{Im}(\sqrt{i})(1-a)|y|^{1 / 2}}\right)}{e^{\operatorname{Im}(\sqrt{i})(1-a)|y|^{1 / 2}}}=O\left(|y|^{-1 / 2}\right) .
$$

This yields $H_{D}(z)=0$; hence $F_{v_{D}}(z)=\left(u_{1,+}-u_{2,+}\right)(a, z)=0$ for all $z \in \mathbb{C}$.

Let $v_{N}(x, z):=v_{2}$ be the solution of (4.3) with the initial conditions $v(a, z)=1$ and $v^{\prime}(a, z)=0$. Then from (4.4) we have

$$
F_{v_{N}}(z)=\left(u_{1,+}^{\prime}-u_{2,+}^{\prime}\right)(a, z) .
$$

Define

$$
H_{N}(z)=\frac{F_{v_{N}}(z)}{G_{S}(z)} .
$$

By the hypothesis (4.1) on $S$ and the fact that $u_{1,+}(a, z)=u_{2,+}(a, z)$, we have from (2.7) and (3.4) that

$$
\begin{aligned}
|H(i y)| & =\frac{\left|m_{+, 1}(a, i y)-m_{+, 2}(a, i y)\right|}{G_{S}(z)}\left|u_{1,+}(a, i y)\right| \\
& \leq \frac{o(1) e^{\operatorname{Im}(\sqrt{i})(1-a)|y|^{1 / 2}}(1+o(1))}{e^{\operatorname{Im}(\sqrt{i})(1-a)|y|^{1 / 2}}} \\
& =o(1) .
\end{aligned}
$$

This gives us that $H_{N}(z)=0$ and $F_{v_{N}}(z)=\left(u_{1,+}^{\prime}-u_{2,+}^{\prime}\right)(a, z)=0$ for all $z \in \mathbb{C}$. Thus, we conclude that $m_{+, 1}(a, z)=m_{+, 2}(a, z)$. By Theorem 2.1, we have $h_{1}=h_{2}$ and $q_{1}=q_{2}$ a.e. on $[a, 1]$ and (hence on $[0,1]$ ). The proof is thus complete. 


\section{THE CASE WHERE $h_{1}=h_{2}$}

In this section, we attack the case where $h_{1}=h_{2}$. In this case we show that it is possible to miss from the spectral data not only one eigenvalue but also some norming constants when $q$ is smooth near $x=0$.

The technique which we use in this section is based on the method discussed by Hochstadt and Hald; see [19, Theorem 1] and [18, Theorem 1.1].

For simplicity, we shall denote by $u_{l, j}(l=1,2)$ the eigenfunctions of the SturmLiouville problems consisting of the equations

$$
u^{\prime \prime}=\left(q_{l}-\lambda_{l, j}\right) u
$$

with the boundary conditions (1.2) and (1.3), and the initial conditions $u(0)=1$ and $u^{\prime}(0)=h_{0}$. In terms of this notation, the norming constants $\kappa_{l, j}=u_{l, j}^{-1}(1)$.

Our main results of this section are the following two theorems.

Theorem 5.1. Let $\Lambda_{0}=\left\{j_{1}, j_{2}, \ldots, j_{k+1}\right\} \subset \mathbb{N}_{0}$ and $\sigma\left(L_{l}\right)=\left\{\lambda_{l, j}\right\}_{j \in \mathbb{N}_{0}}$ for $l=1,2$. Suppose that $q_{1}, q_{2} \in C^{2 k-1}[0, \varepsilon)$ for some $k \in \mathbb{N}$ and for some $\varepsilon>0$. Assume that $h_{1}=h_{2}$,

$$
\begin{gathered}
q_{1}^{(j)}(0)=q_{2}^{(j)}(0), \quad j=0,1, \ldots, 2 k-1, \\
\lambda_{1, j}=\lambda_{2, j}, \quad j \in \mathbb{N}_{0} \backslash\{m\} \quad \text { and } \quad \kappa_{1, j}=\kappa_{2, j}, \quad j \in \mathbb{N}_{0} \backslash \Lambda_{0}
\end{gathered}
$$

with some $m \in \Lambda_{0}$. Then $q_{1}=q_{2}$ a.e. on $[0,1]$.

Theorem 5.2. Let $q_{1}, q_{2} \in L^{1}[0,1]$. Assume that $h_{1}=h_{2}$ and

$$
\lambda_{1, j}=\lambda_{2, j} \quad \text { and } \quad \kappa_{1, j}=\kappa_{2, j}, \quad j \in \mathbb{N} .
$$

Then $q_{1}=q_{2}$ a.e. on $[0,1]$.

Remark 5.3. Comparing the results of Theorems 5.1 and 5.2 with those of [16, Theorem 1.3] and Theorem 3.1 of this present paper, we observe that one can replace information on one eigenvalue by knowledge of the boundary condition $h_{1}$. This shows that the answer to the open problem of Gesztesy and Simon [16] is affirmative under the circumstance of general spectral data.

Before proving Theorems 5.1 and 5.2, we mention a lemma which will be needed subsequently. Its proof is similar to the proofs of [19, Theorem 1] and [18, Theorem $1.1]$ and is therefore omitted.

Lemma 5.4. Let $h_{1}=h_{2}$ and $\Lambda_{0}=\left\{j_{1}, j_{2}, \ldots, j_{k+1}\right\} \subset \mathbb{N}_{0}$. Define the function $\tilde{y}_{j}$ by

$$
\tilde{y}_{j}=\frac{v_{+, j}-\kappa_{1, j} v_{-, j}}{\omega_{1}^{\prime}\left(\lambda_{j}\right)},
$$

where $v_{ \pm, j}$, for each $j$, is the solution of (2.1) with $q=q_{2}$ and $z=\lambda_{1, j}$, and with the initial conditions (2.2)-(2.3). If $\lambda_{1, j}=\lambda_{2, j}$ and $\kappa_{1, j}=\kappa_{2, j}$ for $j \in \mathbb{N}_{0} \backslash \Lambda_{0}$, then

$$
\begin{aligned}
0 & =\sum_{j \in \Lambda_{0}} \tilde{y}_{j}(0), \\
q_{1}-q_{2} & =2 \sum_{j \in \Lambda_{0}}\left(\tilde{y}_{j} u_{1, j}\right)^{\prime} \quad \text { a.e. }
\end{aligned}
$$

Note that if $\lambda_{1, j}=\lambda_{2, j}$ for some $j \in \Lambda_{0}$, then $v_{+, j}=\kappa_{2, j} v_{-, j}$ and $\kappa_{2, j}$ are the eigenfunction and, respectively, the norming constant of the operator $L_{2}$ corresponding to the eigenvalue $\lambda_{2, j}$. 
Proof of Theorem 5.1. We first prove that $\lambda_{1, m}=\lambda_{2, m}$. We have from (5.1) that $\lambda_{1, j}=\lambda_{2, j}$ for all $j \in \mathbb{N}_{0} \backslash\{m\}$. Note that if $j \neq m$, then $v_{-, j}$ and $v_{+, j}$ are the eigenfunctions, corresponding to the eigenvalue $\lambda_{2, j}\left(=\lambda_{1, j}\right)$, of the operator $L_{2}$. In this case, by (5.3) and (2.2)-(2.3), we find $v_{+, j}=\kappa_{2, j} v_{-, j}$ with $\kappa_{2,, j}=v_{+, j}(0)$ and

$$
\tilde{y}_{j}=\frac{v_{+, j}-\kappa_{1, j} v_{-, j}}{\omega_{1}^{\prime}\left(\lambda_{j}\right)}= \begin{cases}\frac{v_{+, j}-\kappa_{1, j} v_{-, j}}{\omega_{1}^{\prime}\left(\lambda_{j}\right)} & \text { if } j=m, \\ \frac{\kappa_{2, j}-\kappa_{1, j}}{\omega_{1}^{\prime}\left(\lambda_{j}\right)} u_{2, j} & \text { if } j \neq m .\end{cases}
$$

Since $q_{1}(0)=q_{2}(0)$ and both $u_{1, j}$ and $u_{2, j}\left(=v_{-, j}\right)$ satisfy the same initial condition at $x=0$, by (5.5) and (5.6) we have

$$
\begin{aligned}
0= & \left(\frac{v_{+, m}-\kappa_{1, m} v_{-, m}}{\omega_{1}^{\prime}\left(\lambda_{m}\right)} u_{1, m}\right)^{\prime}(0)+\sum_{j \in \Lambda_{0} \backslash\{m\}} \frac{\kappa_{2, j}-\kappa_{1, j}}{\omega_{1}^{\prime}\left(\lambda_{j}\right)}\left(u_{1, j} u_{2, j}\right)^{\prime}(0) \\
= & \frac{\left(v_{+, m}^{\prime} u_{1, m}+v_{+, m} u_{1, m}^{\prime}\right)(0)-\kappa_{1, m}\left(v_{-, m}^{\prime} u_{1, m}+v_{-, m} u_{1, m}^{\prime}\right)(0)}{\omega_{1}^{\prime}\left(\lambda_{m}\right)} \\
& +\sum_{j \in \Lambda_{0} \backslash\{m\}}\left(u_{1, j}^{\prime} u_{2, j}+u_{1, j} u_{2, j}^{\prime}\right)(0) \frac{\kappa_{2, j}-\kappa_{1, j}}{\omega^{\prime}\left(\lambda_{j}\right)} \\
= & \frac{v_{+, m}^{\prime}(0)+h_{0} v_{+, m}(0)-2 \kappa_{1, m} h_{0}}{\omega_{1}^{\prime}\left(\lambda_{m}\right)}+2 h_{0} \sum_{j \in \Lambda_{0} \backslash\{m\}} \frac{\kappa_{2, j}-\kappa_{1, j}}{\omega_{1}^{\prime}\left(\lambda_{j}\right)} .
\end{aligned}
$$

By (5.4) and (5.6) we have

$$
0=\sum_{j \in \mathbf{\Lambda}_{0}} \tilde{y}_{j}(0)=\frac{v_{+, m}(0)-\kappa_{m}}{\omega_{1}^{\prime}\left(\lambda_{m}\right)}+\sum_{j \in \boldsymbol{\Lambda}_{0} \backslash\{m\}} \frac{\kappa_{2, j}-\kappa_{1, j}}{\omega_{1}^{\prime}\left(\lambda_{j}\right)} .
$$

Substituting (5.8) into (5.7), we get

$$
\frac{v_{+, m}^{\prime}(0)-h_{0} v_{+, m}(0)}{\omega_{1}^{\prime}\left(\lambda_{m}\right)}=0 .
$$

This shows that $\lambda_{1, m}$ is an eigenvalue of the operator $L_{2}$. If $\lambda_{1, m} \neq \lambda_{2, m}$, then we may assume without loss of generality that $\lambda_{1, m}<\lambda_{2, m}$. Thus $\lambda_{1, m}<\lambda_{2, m}<$ $\lambda_{1, m+1}=\lambda_{2, m+1}$ and

$$
\lambda_{2,0}<\lambda_{2,1}<\cdots<\lambda_{2, m}<\lambda_{1, m}<\lambda_{2, m+1} \cdots .
$$

But this contradicts the asymptotic result (see [12, p. 5]) for the eigenvalues of the operator $L_{2}$. Hence $\lambda_{1, m}=\lambda_{2, m}$, and we get $\lambda_{1, j}=\lambda_{2, j}$ for all $j \in \mathbb{N}_{0}$.

Based on the above result (that is, $\lambda_{1, j}=\lambda_{2, j}$ for all $j \in \mathbb{N}_{0}$ ), we next prove that $\kappa_{1, j}=\kappa_{2, j}$ for all $j \in \Lambda_{0}$. Write $\lambda_{1, j}\left(=\lambda_{2, j}\right)$ as $\lambda_{j}$, and without loss of generality we assume that $\Lambda_{0}=\{0,1, \ldots, k\}$. Set $A_{j}:=\left(\kappa_{1, j}-\kappa_{2, j}\right) / \omega^{\prime}\left(\lambda_{j}\right)$. It follows from (5.6) that $\tilde{y}_{j}=A_{j} u_{2, j}$. Hence (5.5) can be rewritten as

$$
\left(q_{1}-q_{2}\right)(x)=2 \sum_{j=0}^{k} A_{j}\left(u_{1, j} u_{2, j}\right)^{\prime}(x) \quad \text { for } x \in[0,1] .
$$

It is easy to observe that $q_{1}-q_{2} \in C^{2 k}[0, \varepsilon)$, since $u_{i, j}$ are the solutions of (2.1) for $q=q_{i}, i=1,2$. We next show that

$$
\sum_{j=0}^{k} \lambda_{j}^{l} A_{j}=0, \quad \text { for all } l=0,1, \ldots, k .
$$


We use induction to prove this fact. It is easy to check that if $l=0,1$, then (5.10) follows from (5.4) and the following computation:

$$
\begin{aligned}
0 & =1 / 2\left(q_{1}^{\prime}(0)-q_{2}^{\prime}(0)\right) \\
& =\sum_{j=0}^{k} A_{j}\left(u_{1, j} u_{2, j}\right)^{\prime \prime}(0) \\
& =\sum_{j=0}^{k} A_{j}\left[\left(u_{1, j}^{\prime \prime} u_{2, j}+u_{1, j} u_{2, j}^{\prime \prime}\right)+2 u_{1, j}^{\prime} u_{2, j}^{\prime}\right](0) \\
& =\sum_{j=0}^{k} A_{j}\left[\left(q_{1}(0)+q_{2}(0)-2 \lambda_{j}\right)\left(u_{1, j} u_{2, j}\right)(0)+2\left(u_{1, j}^{\prime} u_{2, j}^{\prime}\right)(0)\right] \\
& =\left(q_{1}(0)+q_{2}(0)+2 h_{0}^{2}\right) \sum_{j=0}^{k} A_{j}-2 \sum_{j=0}^{k} \lambda_{j} A_{j} .
\end{aligned}
$$

Now suppose that (5.10) holds for $l=0,1, \ldots, t(<k)$. We shall verify that (5.10) also holds for $l=t+1$. Note that $q_{1}, q_{2} \in C^{2 k-1}[0, \varepsilon)$ for some $\varepsilon>0$ and $\left(q_{1}-q_{2}\right)^{(j)}(0)=$ 0 for $j=0,1, \ldots, 2 k-1$. Differentiating the identity (5.5) $(2 t+1)$ times with respect to $x$ and using (2.1) and its coefficients, we find that, by letting $x=0$,

$$
\begin{aligned}
0= & 1 / 2\left(q_{1}-q_{2}\right)^{(2 t+1)}(0) \\
= & \sum_{j=0}^{k} A_{j}\left(u_{1, j} u_{2, j}\right)^{(2 t+2)}(0) \\
= & \sum_{j=0}^{k} A_{j}\left[\left(u_{1, j}^{\prime \prime} u_{2, j}+u_{1, j} u_{2, j}^{\prime \prime}\right)+2 u_{1, j}^{\prime} u_{2, j}^{\prime}\right]^{(2 t)}(0) \\
= & -2 \sum_{j=0}^{k} \lambda_{j} A_{j}\left(u_{1, j} u_{2, j}\right)^{(2 t)}(0)+\sum_{j=0}^{k} A_{j}\left[\left(q_{1}+q_{2}\right)\left(u_{1, j} u_{2, j}\right)\right]^{(2 t)}(0) \\
& +\sum_{j=0}^{k} 2 A_{j}\left(u_{1, j}^{\prime} u_{2, j}^{\prime}\right)^{(2 t)}(0) .
\end{aligned}
$$

Next, we calculate $u_{1, j}^{(l)}(0)$ and $u_{2, j}^{(l)}(0)$ for $l=0,1,2, \ldots, 2 t$. Note that

$$
\begin{aligned}
u_{1, j}(0)= & 1, \quad u_{1, j}^{\prime}(0)=h_{0}, \quad u_{1, j}^{\prime \prime}(0)=q_{1}(0)-\lambda_{j}, \\
u_{1, j}^{\prime \prime \prime}(0)= & \left(q_{1}^{\prime} u_{1, j}\right)(0)+\left(\left(q_{1}-\lambda_{j}\right) u_{1, j}^{\prime}\right)(0) \\
= & q_{1}^{\prime}(0)+q_{1}(0) h_{0}-h_{0} \lambda_{j}, \\
u_{1, j}^{(4)}(0)= & \left(q_{1}^{\prime \prime} u_{1, j}\right)(0)+2\left(q_{1}^{\prime} u_{1, j}^{\prime}\right)(0)+\left(\left(q_{1}-\lambda_{j}\right)^{2} u_{1, j}\right)(0) \\
= & \left(q_{1}^{\prime \prime}+q_{1}^{2}+2 h_{0} q_{1}^{\prime}\right)(0)-2 q_{1}(0) \lambda_{j}+\lambda_{j}^{2}, \\
u_{1, j}^{(5)}(0)= & {\left[\left(q_{1}^{\prime \prime}+q_{1}^{2}-2 \lambda_{j} q_{1}\right) u_{1, j}\right]^{\prime}(0)+2\left(q_{1}^{\prime} u_{1, j}^{\prime}\right)^{\prime}(0)+\lambda_{j}^{2} u_{1, j}^{\prime}(0) } \\
= & \left(q_{1}^{\prime \prime \prime}+3 q_{1}^{\prime \prime}+3 q_{1} q_{1}^{\prime}+\left(1+h_{0}\right) q_{1}^{2}\right)(0) \\
& -2\left(2 q_{1}^{\prime}-2 h_{0} q_{1}\right)(0) \lambda_{j}+h_{0} \lambda_{j}^{2} .
\end{aligned}
$$


Proceeding by induction, we obtain that $u_{1, j}^{(l)}(0)$ and $u_{2, j}^{(l)}(0)$ for $l=2,3, \ldots, 2 t$ can be expressed as the following forms, for $l=2 s-1$ and $2 s$, respectively:

$$
\begin{aligned}
u_{1, j}^{(2 s-1)}(0) & =(-1)^{s-1} h_{0} \lambda_{j}^{s-1}+a_{s-2} \lambda_{j}^{s-2}+a_{s-3} \lambda_{j}^{s-3}+\cdots+a_{0}, \\
u_{1, j}^{(2 s)}(0) & =(-1)^{s} \lambda_{j}^{s}+b_{s-1} \lambda_{j}^{s-1}+b_{s-3} \lambda_{j}^{s-2}+\cdots+b_{0},
\end{aligned}
$$

where all the coefficients $a_{l}$ and $b_{l}$ of $\lambda_{j}^{l}$ depend only on $h_{0}, q_{1}(0), q_{1}^{\prime}(0), \ldots, q_{1}^{(2 t-2)}(0)$. We observe that $u_{1, j}^{(2 s)}(0)$ is a polynomial of $\lambda_{j}$ with degree $s$ and that $u_{1, j}^{(2 s-1)}(0)$ is also a polynomial of $\lambda_{j}$ with degree at most $s-1$. By (5.12), condition (5.1) and the fact that $\sigma\left(L_{1}\right)=\sigma\left(L_{2}\right)$, we obtain that $u_{1, j}^{(l)}(0)=u_{2, j}^{(l)}(0)$. Thus, using Leibnitz's formula, we have

$$
\begin{aligned}
\left(u_{1, j} u_{2, j}\right)^{(2 t)}(0)= & \sum_{n=0}^{2 t} C_{2 t}^{n}\left(u_{1, j}^{(n)} u_{2, j}^{(2 t-n)}\right)(0) \\
= & \sum_{n=0}^{t} C_{2 t}^{2 n}\left(u_{1, j}^{(2 n)} u_{2, j}^{(2 t-2 n)}\right)(0) \\
& +\sum_{n=1}^{t} C_{2 t}^{2 n-1}\left(u_{1, j}^{(2 n-1)} u_{2, j}^{(2 t-2 n+1)}\right)(0) \\
= & (-1)^{t}\left(\sum_{n=0}^{t} C_{2 t}^{2 n}\right) \lambda_{j}^{t}+(-1)^{t-1} h_{0}^{2}\left(\sum_{n=1}^{t} C_{2 t}^{2 n-1}\right) \lambda_{j}^{t-1} \\
& +c_{t-2} \lambda_{j}^{t-2}+c_{t-3} \lambda_{j}^{t-3}+\cdots+c_{0}
\end{aligned}
$$

and

$$
\begin{aligned}
\left(u_{1, j}^{\prime} u_{2, j}^{\prime}\right)^{(2 t)}(0)= & \sum_{n=0}^{2 t} C_{2 t}^{n}\left(u_{1, j}^{(n+1)} u_{2, j}^{(2 t-n+1)}\right)(0) \\
= & (-1)^{t} h_{0}^{2}\left(\sum_{n=0}^{t} C_{2 t}^{2 n}\right) \lambda_{j}^{t}+(-1)^{t} h_{0}\left(\sum_{n=1}^{t} C_{2 t}^{2 n-1}\right) \lambda_{j}^{t} \\
& +c_{t-1}^{\prime} \lambda_{j}^{t-1}+c_{t-2}^{\prime} \lambda_{j}^{t-2}+\cdots+c_{0}^{\prime},
\end{aligned}
$$

where $C_{2 t}^{n}=2 t(2 t-1) \cdots(2 t-n+1) / n$ ! and all the coefficients $c_{n}$ and $c_{n}^{\prime}$ depend only on $h_{0}, q_{1}(0), q_{1}^{\prime}(0), \ldots, q_{1}^{(2 t-2)}(0)$.

Similarly, using Leibnitz's formula again we easily obtain that

$$
\left[\left(q_{1}+q_{2}\right)\left(u_{1, j} u_{2, j}\right)\right]^{(2 t)}(0)=2 \sum_{n=0}^{2 t} C_{2 t}^{n} q_{1}^{(n)}(0)\left(u_{1, j} u_{2, j}\right)^{(2 t-n)}(0)
$$

is a polynomial of $\lambda_{j}$ with degree at most $t$ for which the coefficients are only associated with $h_{0}$ and $q_{1}^{(n)}(0)\left(=q_{2}^{(n)}(0)\right)$ for $0 \leq n \leq 2 t$.

By the relations (5.13)-(5.15), the identity (5.11) can be rewritten as the form

$$
\begin{aligned}
0 & =\sum_{j=0}^{k} A_{j}\left(u_{1, j} u_{2, j}\right)^{(2 t+2)}(0) \\
& =\sum_{j=0}^{k} 2(-1)^{t+1}\left(\sum_{n=0}^{t} C_{2 t}^{2 n}\right) \lambda_{j}^{t+1} A_{j}+\sum_{j=0}^{k} c_{t, j} \lambda_{j}^{t} A_{j}+\cdots+\sum_{j=0}^{k} c_{0, j} A_{j},
\end{aligned}
$$


where the coefficients $c_{i, j}$ for $i=0, \ldots, t$ and $j=0, \ldots, k$ are the linear combinations of the coefficients of polynomials (5.13)-(5.15). It turns out from the presupposition that (5.10) holds for $l=t+1$ and, hence, for $l=0, \ldots, k$.

By the fact that $\lambda_{0}<\lambda_{1}<\cdots<\lambda_{k}$ and the fact that the Vandermonde determinant associating with $\left\{\lambda_{j}\right\}_{j=0}^{k}$ does not vanish, we conclude that $A_{j}=0$ from (5.10) and therefore $\kappa_{1, j}=\kappa_{2, j}$ for all $j \in \Lambda_{0}$. By (5.9) we further conclude that $q_{1}=q_{2}$ a.e. on $[0,1]$. The proof is therefore complete.

Next, we prove Theorem 5.2.

Proof of Theorem 5.2. All we need to prove is that $\lambda_{1,0}=\lambda_{2,0}$ and $\kappa_{1,0}=\kappa_{2,0}$. Suppose on the contrary that $\lambda_{1,0} \neq \lambda_{2,0}$; then $\tilde{y}_{0} \not \equiv 0$ on $[0,1]$ (see (5.3) for the definition of $\left.\tilde{y}_{0}\right)$. By the asymptotic expansions of $\lambda_{i, n}$,

$$
\lambda_{i, n}=(n \pi)^{2}+2\left(h_{i}-h_{0}\right)+\int_{0}^{1} q_{i}(t) d t+o(1), \quad i=1,2,
$$

and the assumptions that $h_{1}=h_{2}$ and $\lambda_{1, n}=\lambda_{2, n}$ for all $n \in \mathbb{N}$, we immediately get

$$
\int_{0}^{1}\left(q_{1}-q_{2}\right)(t) d t=0
$$

Moreover, we have, by $(5.4), \tilde{y}_{0}(0)=0$ and, by $(5.5)$,

$$
0=\int_{0}^{1}\left(q_{1}-q_{2}\right)(t) d t=\tilde{y}_{0}(1) u_{-, 0}(1)=\tilde{y}_{0}(1) .
$$

This shows that $\lambda_{1,0}$ is an eigenvalue of (2.1) with $q=q_{2}$ and the Dirichlet boundary conditions, i.e., $u(0)=0=u(1)$. By [36, Chapter 4] we have $\lambda_{1,0}>\lambda_{2,0}$. If we interchange the positions of $q_{1}$ and $q_{2}$ in Lemma 5.4, then, by repeating the argument above, we get $\lambda_{1,0}<\lambda_{2,0}$. This contradicts the assumption that $\tilde{y}_{0} \not \equiv 0$. Consequently, we must have $\lambda_{1,0}=\lambda_{2,0}$ which together with (5.4) implies that $\kappa_{1,0}=\kappa_{2,0}$. Moreover, by (5.5), we have $q_{1}=q_{2}$ on $[0,1]$.

\section{Dirichlet Boundary CONDITION}

In this section, we shall provide some details in the remaining cases, for which the operators $L$ in $L^{2}[0,1]$ involve the Dirichlet boundary conditions at $x=0$ and/or $x=1$. More precisely, we shall consider some analogs to Theorem 3.4 and Theorem 4.1 in the Dirichlet context. We need to distinguish three cases:

Case I. $L$ has a Dirichlet boundary condition at $x=0$ and $x=1$, that is,

$$
u(0)=0=u(1) .
$$

Case II. $L$ has a non-Dirichlet boundary condition at $x=0$ and a Dirichlet boundary condition at $x=1$, that is,

$$
u^{\prime}(0)-h_{0} u(0)=0=u(1), \quad h_{0} \in \mathbb{R} .
$$

Case III. $L$ has a Dirichlet boundary condition at $x=0$ and a non-Dirichlet boundary condition at $x=1$, that is,

$$
u(0)=0=u^{\prime}(1)-h_{1} u(1), \quad h_{0} \in \mathbb{R} .
$$

We index the corresponding eigenvalues $\lambda_{j}$ of the operator $L$ by

$$
\left\{\lambda_{j}\right\}_{j=1}^{\infty} \text { in Case I and }\left\{\lambda_{j}\right\}_{j=0}^{\infty} \quad \text { in Cases II and III. }
$$


Let $u_{1,+}(x, z)$ and $u_{2,+}(x, z)$ be the solutions of (2.1) corresponding to $q_{1}$ and $q_{2}$, respectively, where $u_{j,+}(x, z)$ satisfies the boundary conditions at $x=1$ and normalization, for $j=1,2$,

$$
\begin{array}{lll}
u_{j,+}(1, z)=0, & u_{j,+}^{\prime}(1, z)=1 & \text { in Cases I and II, } \\
u_{j,+}(1, z)=1, & u_{j,+}^{\prime}(1, z)=h_{1} & \text { in Case III. }
\end{array}
$$

Note that the asymptotic expansions of $u_{j,+}$ and $u_{j,+}^{\prime}$ are given in (3.2) and (3.3) in Case III, and in Cases I and II we have

$$
\begin{aligned}
& u_{j,+}(x, z)=-\frac{\sin (\sqrt{z}(1-x))}{\sqrt{z}}+O\left(\frac{e^{\operatorname{Im}(\sqrt{z})(1-x)}}{z}\right), \\
& u_{j,+}^{\prime}(x, z)=\cos (\sqrt{z}(1-x))+O\left(\frac{e^{\operatorname{Im}(\sqrt{z})(1-x)}}{\sqrt{z}}\right)
\end{aligned}
$$

as $|z| \rightarrow \infty$ for all $x \in[0,1]$. When $a \in[0,1)$ and if $z=i y$ with $y \in \mathbb{R}$, then (6.7) and (6.8) imply

$$
\begin{aligned}
\left|u_{j,+}(a, i y)\right| & =\frac{1}{2}|y|^{-1 / 2} e^{\operatorname{Im}(\sqrt{i})(1-a)|y|^{1 / 2}}(1+o(1)), \\
\left|u_{j,+}^{\prime}(a, i y)\right| & =\frac{1}{2} e^{\operatorname{Im}(\sqrt{i})(1-a)|y|^{1 / 2}}(1+o(1))
\end{aligned}
$$

as $y \rightarrow \infty$. For $j=1,2$, let

$$
\begin{aligned}
& \omega_{j}(z)=u_{j,+}(0, z) \quad \text { in Cases I and III, } \\
& \omega_{j}(z)=u_{j,+}^{\prime}(0, z)-h_{0} u_{j,+}(0, z) \quad \text { in Case II. }
\end{aligned}
$$

Then $\sigma\left(L_{j}\right)=\left\{\lambda_{j, n}\right\}_{n=1}^{\infty}$ in Case I, and $\sigma\left(L_{j}\right)=\left\{\lambda_{j, n}\right\}_{n=0}^{\infty}$ in Cases II and III are precisely the set of zeros of the characteristic functions $\omega_{j}(z)$. By (6.9) and (6.10) we also infer that, as $y$ (real) $\rightarrow \infty$,

$$
\begin{aligned}
\left|\omega_{j}(i y)\right| & =\frac{1}{2}|y|^{-1 / 2} e^{\operatorname{Im}(\sqrt{i})|y|^{1 / 2}}(1+o(1)) & & \text { in Case I, } \\
\left|\omega_{j}(i y)\right| & =\frac{1}{2} e^{\operatorname{Im}(\sqrt{i})|y|^{1 / 2}}(1+o(1)) & & \text { in Cases II and III }
\end{aligned}
$$

and for sufficiently large $n$,

$$
\begin{array}{ll}
\inf _{\theta \in[0,2 \pi]} \mid \omega_{j}\left(\left(\left(\pi\left(n+\frac{1}{2}\right)\right)^{2} e^{i \theta}\right) \mid \geq \frac{1}{\pi n}+O\left(\frac{1}{n^{2}}\right)\right. & \text { in Case I, } \\
\inf _{\theta \in[0,2 \pi]} \mid \omega_{j}\left(\left((\pi(n+1))^{2} e^{i \theta}\right) \mid \geq 1+O\left(\frac{1}{n}\right)\right. & \text { in Cases II and III. }
\end{array}
$$

It is easy to see that the norming constants $\kappa_{j, n}$ for $j=1,2$ can be expressed as

$$
\begin{array}{ll}
\kappa_{j, n}=u_{j,+}^{\prime}\left(0, \lambda_{j, n}\right) & \text { in Cases I and III, } \\
\kappa_{j, n}=u_{j,+}\left(0, \lambda_{j, n}\right) & \text { in Case II, }
\end{array}
$$

where $n \in \mathbb{N}$ in Case I and $n \in \mathbb{N}_{0}$ in Cases II and III.

Based upon the above preliminaries, we give an analog of Theorem 3.3 in all of the Cases I, II and III.

Theorem 6.1. Let the operators $L_{1}$ and $L_{2}$ be with one of the boundary conditions (6.1), (6.2), or (6.3). Let $a \in[0,1 / 2)$ and $q_{1}, q_{2} \in C^{n}(a-\varepsilon, a+\varepsilon)$ for some $n \in \mathbb{N}_{0}$ 
and for some $\varepsilon>0$. Consider a subset $S \subseteq \sigma\left(L_{2}\right)$ such that $\kappa_{1, j}=\kappa_{2, j}$ for all $\lambda_{j} \in S$. Assume that $q_{1}=q_{2}$ a.e. on $[0, a], \sigma\left(L_{1}\right)=\sigma\left(L_{2}\right)$ and

$$
\begin{array}{ll}
n_{S}(t) \geq(1-2 a) n_{\sigma\left(L_{2}\right)}(t)-a-\frac{n+1}{2} & \text { in Case I, } \\
n_{S}(t) \geq(1-2 a) n_{\sigma\left(L_{2}\right)}(t)-\frac{n+3}{2} & \text { in Case II, } \\
n_{S}(t) \geq(1-2 a) n_{\sigma\left(L_{2}\right)}(t)-\frac{n+1}{2} & \text { in Case III, }
\end{array}
$$

for all sufficiently large $t \in \mathbb{R}$. Then $h_{1}=h_{2}$ (i.e., $h_{1}=\infty$ in Cases $I$ and II and $h_{1} \in \mathbb{R}$ in Case III) and $q_{1}=q_{2}$ a.e. on $[0,1]$.

Proof. Consider the function defined by

$$
F(z):=\left(u_{1,+}(0, z)-u_{2,+}(0, z)\right) \widetilde{\omega}(z),
$$

where $\widetilde{\omega}(z)=v_{2,+}^{\prime}(0, z)-h_{0} v_{2,+}(0, z)$ and where $v_{2,+}(x, z)$ is the solution of $(2.1)$ for $q_{2}$ with the initial condition (6.6) in Cases I and II or the initial condition (6.5) in Case III. Note that in Cases I and III (i.e., $\left.h_{0}=\infty\right), \widetilde{\omega}(z)=v_{2,+}(0, z)$. Thus, by (3.25) and Green's formula (2.14), we get

$$
\begin{aligned}
F(z) & =\left[u_{1,+}, v_{2,+}\right](a, z)-\left[u_{2,+}, v_{2,+}\right](a, z) \\
& =u_{1,+}(a, z) v_{2,+}(a, z)\left(m_{+, v_{2,+}}(a, z)-m_{+, u_{1,+}}(a, z)\right)-C_{0},
\end{aligned}
$$

where $C_{0}=\left[u_{2,+}, v_{2,+}\right](1, z)=\left(u_{2,+} v_{2,+}^{\prime}\right)(1, z)-\left(u_{2,+}^{\prime} v_{2,+}\right)(1, z)$ is independent of $z$ by the initial conditions. Without loss of generality we may assume that the initial condition for $u_{1,+}$ is the Dirichlet condition in Cases I and II and do not assume a priori that $u_{2,+}$ has the Dirichlet condition at $x=1$. Note that, in all the cases, one of the initial conditions at $x=1$ for $u_{1,+}$ and $v_{2,+}$ is the Dirichlet condition and the other one is a non-Dirichlet condition. Then by (2.8), (3.4) and (6.9) we infer

$$
\begin{aligned}
|F(i y)| & \leq|y|^{-1 / 2} e^{\operatorname{Im}(\sqrt{i}) 2(1-a)|y|^{1 / 2}}(1+o(1)) o\left(|y|^{-(n+1) / 2}\right)+\left|C_{0}\right| \\
& \leq|y|^{-(n+2) / 2} e^{\operatorname{Im}(\sqrt{i}) 2(1-a)|y|^{1 / 2}} o(1) .
\end{aligned}
$$

Following the argument employed in the proof of Theorem 3.4, we again introduce the functions

$$
\begin{aligned}
& G_{S}(z) \quad=\prod_{\lambda_{j} \in S}\left(1-\frac{z}{\lambda_{j}}\right) \quad S \subseteq \sigma\left(L_{1}\right), \\
& G_{\sigma\left(L_{1}\right)}(z)=\prod_{j=1}^{\infty}\left(1-\frac{z}{\lambda_{j}}\right) \quad \text { in Case I, } \\
& G_{\sigma\left(L_{1}\right)}(z)=\prod_{j=0}^{\infty}\left(1-\frac{z}{\lambda_{j}}\right) \quad \text { in Cases II and III. }
\end{aligned}
$$

Then from (6.19)-(6.21) and the proof of Theorem 3.4 we have

$$
\left.\begin{array}{ll}
\left|G_{S}(i y)\right| \geq C_{0}|y|^{-(n+2) / 2} e^{\operatorname{Im}(\sqrt{i})(1-2 a)|y|^{1 / 2}} & \text { in Case I, } \\
\left|G_{S}(i y)\right| \geq C_{0}|y|^{-(n+3) / 2} e^{\operatorname{Im}(\sqrt{i})(1-2 a)|y|^{1 / 2}} & \text { in Case II, } \\
\left|G_{S}(i y)\right| \geq C_{0}|y|^{-(n+1) / 2} e^{\operatorname{Im}(\sqrt{i})(1-2 a)|y|^{1 / 2}} & \text { in Case III. }
\end{array}\right\}
$$


By the definition of $\widetilde{\omega}(\cdot)$ and the boundary conditions of the solution $v_{2}$, we deduce that

$$
\left.\begin{array}{rlrl}
|\widetilde{\omega}(i y)| & =\frac{1}{2} e^{\operatorname{Im}(\sqrt{i})|y|^{1 / 2}}(1+o(1)) & & \text { in Case I, } \\
|\widetilde{\omega}(i y)| & =\frac{1}{2}|y|^{1 / 2} e^{\operatorname{Im}(\sqrt{i})|y|^{1 / 2}}(1+o(1)) & & \text { in Case II, } \\
|\widetilde{\omega}(i y)| & =\frac{1}{2}|y|^{-1 / 2} e^{\operatorname{Im}(\sqrt{i})|y|^{1 / 2}}(1+o(1)) & & \text { in Case III. }
\end{array}\right\}
$$

Let $G(z)=G_{S}(z) \widetilde{\omega}(z)$. By (6.12)-(6.14), we find that

$$
|G(i y)| \geq C_{0}|y|^{-(n+2) / 2} e^{\operatorname{Im}(\sqrt{i}) 2(1-a)|y|^{1 / 2}} .
$$

Now using (6.24) and (6.28), one can complete the proof of Theorem 6.1 by repeating the proof of Theorem 3.4 in Section 3.

Remark 6.2. If $q$ is assumed to be $C^{2 k-1}[0, \varepsilon)$, then $q^{(j)}(0), j=0,1, \ldots, 2 k-1$, together with the knowledge of $h_{0} \in \overline{\mathbb{R}}$, and all the eigenvalues of $L$ and all the corresponding norming constants except for $k$ in Cases I and III, and for $(k+1)$ in Case II uniquely determine $h_{1}$ and $q$ on all of $[0,1]$. In particular, if $q \in L^{1}[0,1]$, then one norming constant can be missing in Case II.

Finally, we give an analog of Theorem 4.1 in the Dirichlet context.

Theorem 6.3. Let the operators $L_{1}$ and $L_{2}$ be with one of the boundary conditions (6.1), (6.2), or (6.3). Let $a \in[0,1)$. Consider a subset $S \subseteq \sigma\left(L_{1}\right) \cap \sigma\left(L_{2}\right)$ such that $\kappa_{1, j}=\kappa_{2, j}$ for all $\lambda_{j} \in S$. Assume that $q_{1}=q_{2}$ a.e. on $[0, a]$ and

$$
\begin{array}{lll}
n_{S}(t) \geq(1-a) n_{\sigma\left(L_{2}\right)}(t)-\frac{a+1}{2} & & \text { in Case I, } \\
n_{S}(t) \geq(1-2 a) n_{\sigma\left(L_{2}\right)}(t)-\frac{1}{2} & & \text { in Case II. } \\
n_{S}(t) \geq(1-2 a) n_{\sigma\left(L_{2}\right)}(t) & & \text { in Case III }
\end{array}
$$

for all sufficiently larget. Then $h_{1}=h_{2}$ (i.e., $h_{1}=\infty$ in Cases $I$ and II and $h_{1} \in \mathbb{R}$ in Case III) and $q_{1}=q_{2}$ a.e. on $[0,1]$.

Proof. Let us consider the following function:

$$
F_{v}(z):=\left(h_{1}-h_{2}\right) v_{2}(1, z)+\int_{0}^{1}\left(q_{1}-q_{2}\right)(x)\left(u_{1,+} v_{2}\right)(x, z) d x .
$$

Let $v_{D}:=v_{2}(x, z)$ (resp., $v_{N}:=v_{2}(x, z)$ ) be the solution of (2.1) for $q_{2}$ satisfying the initial condition (6.5) (resp., (6.6) with $h_{1}=0$ ) at point $x=a$, respectively. By virtue of the proof of Theorem 4.1, we have

$$
F_{v_{D}}(z)=\left(u_{1,+}-u_{2,+}\right)(a, z), \quad F_{v_{N}}(z)=\left(u_{1,+}^{\prime}-u_{2,+}^{\prime}\right)(a, z) .
$$

Since $h_{0}$ is fixed and there is an infinite sequence of common eigenvalues of the operators $L_{1}$ and $L_{2}$ (see (6.29)-(6.31)), and by the asymptotic expansions of $\left\{\lambda_{j, n}\right\}$ for $j=1,2$,

$$
\begin{array}{ll}
\sqrt{\lambda_{j, n}}=n \pi+O\left(\frac{1}{n}\right) & \text { in Case I, } \\
\sqrt{\lambda_{j, n}}=\left(n+\frac{1}{2}\right) \pi+O\left(\frac{1}{n}\right) & \text { in Cases II and III }
\end{array}
$$


as $n \rightarrow \infty$, we infer that the initial conditions for $u_{1,+}$ and $u_{2,+}$ at $x=1$ are of the same type, that is, both initial conditions are the Dirichlet condition in Cases I and II and in the non-Dirichlet condition in Case III. This ensures that $h_{1}=h_{2}=\infty$ in Cases I and II and $h_{1}, h_{2} \in \mathbb{R}$ in Case III. Thus, by (6.7) and (3.2) we have

$$
\left.\begin{array}{ll}
\left|F_{v_{D}}(i y)\right|=O\left(|y|^{-1} e^{\left.\operatorname{Im}(\sqrt{i})(1-a)|y|^{1 / 2}\right)}\right. & \text { in Cases I and II, } \\
\left|F_{v_{D}}(i y)\right|=O\left(|y|^{-1 / 2} e^{\left.\operatorname{Im}(\sqrt{i})(1-a)|y|^{1 / 2}\right)}\right. & \text { in Case III. }
\end{array}\right\}
$$

Following the argument employed in the proof of Theorem 4.1, we again introduce the function

$$
G_{S}(z)=\prod_{\lambda_{j} \in S}\left(1-\frac{z}{\lambda_{j}}\right), \quad S \subseteq \sigma\left(L_{2}\right) .
$$

By the hypotheses (6.29)-(6.31) on $S$ we have

$$
\left.\begin{array}{ll}
\left|G_{S}(i y)\right| \geq C_{0}|y|^{-1 / 2} e^{\operatorname{Im}(\sqrt{i})(1-a)|y|^{1 / 2}} & \text { in Cases I and II, } \\
\left|G_{S}(i y)\right| \geq C_{0} e^{\left.\operatorname{Im}(\sqrt{i})(1-a)|y|^{1 / 2}\right)} & \text { in Case III. }
\end{array}\right\}
$$

This yields that $F_{v_{D}}(z)=\left(u_{1,+}-u_{2,+}\right)(a, z)=0$ for all $z \in \mathbb{C}$. In this case,

$$
F_{v_{N}}(z)=\left(m_{+, 1}(a, z)-m_{+, 2}(a, z)\right) u_{1,+}(a, z) .
$$

Using (2.7) and (6.9) we can complete the proof of Theorem 6.3 by following the same method as that of Theorem 4.1 in Section 4.

\section{ACKNowledgement}

The research of the first author was supported in part by the NNSF of China (No. 10771165), and the research of the second author was supported in part by NSC 97-2628-M-110-003-MY3 (Taiwan). Both authors are grateful to the referees for their helpful comments and suggestions which improved and strengthened the presentation of this paper.

\section{REFERENCES}

[1] Atkinson, F.V., On the location of the Weyl Circles, Proc. Roy. Soc. Edinburgh 88A, 1981, pp. 345-356. MR616784 (83a:34023)

[2] Amour, L., and Raoux,T., Inverse spectral results for Schrödinger operators on the unit interval with potentials in $L_{p}$ spaces, Inverse Probl. 23, 2007, pp. 23-67. MR 2441008|(2009f:34220)

[3] Amour, L., and Raoux,T., Inverse spectral results for Schrödinger operators on the unit interval with partial information given on the potentials, J. Math Phy., 50, 2009, 033505, pp. 1-14. MR2510910 (2010g:81301)

[4] Borg, G., Eine Umkehrung der Sturm-Liouvilleschen Eigenwertaufgabe, Acta Math., 78, 1946, pp. 1-96. MR0015185(7:382d)

[5] Borg, G., Uniqueness theorems in the spectral theory of 0, Proc. 11th Scandinavian Congr. Math. (Trondheim, 1949), Johan Grundt Tanums Forlag, Oslo, 1952, pp. 276-287. MR0058063 (15:315a)

[6] Coleman, C.F., and McLaughlin, J.R., Solution of the inverse spectral problem for an impedance with integrable derivative. I, Comm. Pure Appl. Math., 46, 1993, pp. 145-184. MR.1199197 (93m:34017)

[7] Coleman, C.F., and McLaughlin,J.R., Solution of the inverse spectral problem for an impedance with integrable derivative. II, Comm. Pure Appl. Math., 46, 1993, pp. 185-212. MR1199197 (93m:34017)

[8] Dahlberg, B., and Trubowitz, E., The inverse Sturm-Liouville problem. III. Comm. Pure Appl. Math., 37, 1984, pp. 255-267. MR733718 (86f:34052) 
[9] Danielyan, A., and Levitan, B.M., On the asymptotic behavior of the Weyl-Titchmarsh mfunction, Math. USSR Izv., 36, 1991, pp. 487-496. MR1072691 (92d:34056)

[10] del Rio, R., Gesztesy, F., and Simon, B., Inverse spectral analysis with partial information on the potential, III. Updating boundary conditions, Internat. Math. Res. Notices 15, 1997, pp. 751-758. MR1470376 (99a:34032)

[11] Everitt, W.N., On a property of the m-coeffcient of a second-order linear differential equation, J. London Math. Soc., 4, 1972, pp. 443-457. MR0298104 (45:7156)

[12] Freiling, G., and Yurko, V., Inverse Sturm-Liouville Problems and Their applications, Nova Science Publishers Inc., Huntington, 2001. MR2094651 (2005f:34001)

[13] Gesztesy, F., and Simon B., m-functions and inverse spectral analysis for finite and semiinfinite Jacobi matrices, J. d'Analyse Math., 73, 1997, pp. 267-297. MR.1616422 (99c:47039)

[14] Gesztesy, F., and Simon B., Uniqueness theorems in inverse spectral theory for onedimensional Schrödinger operators, Trans. Amer. Math. Soc., 348, 1996, pp. 349-373. MR.1329533 (96e:34030)

[15] Gesztesy, F., and Simon B., Inverse spectral analysis with partial information on the potential, I. The case of an a.c. component in the spectrum, Helv. Phys. Acta, 70, 1997, pp. 66-71. MR.1441597 (98f:81347)

[16] Gesztesy, F., and Simon B., Inverse spectral analysis with partial information on the potential, II. the case of discrete spectrum, Trans. Amer. Math. Soc., 352, 2000, pp. 2765-2787. MR:1694291 (2000j:34019)

[17] Hald, O.H. Inverse eigenvalue problem for the mantle, Geophys. J. R. Astr. Soc., 62, 1980, pp. 41-48.

[18] Hald, O.H., The Sturm-Liouville problem with symmetric potentials, Acta Math., 141, 1978, pp. 263-291. MR505878 (80a:34022)

[19] Hochstadt, H., The inverse Sturm-Liouville problem, Commun. Pure Appl. Math., 26, 1973, pp. 715-729. MR0330607 (48:8944)

[20] Hochstadt, H., and Lieberman, B., An inverse Sturm-Liouville problem with mixed given data, SIAM J. Appl. Math., 34, 1978, pp. 676-680. MR0470319 (57:10077)

[21] Isaacson, E., and Trubowitz, E., The inverse Sturm-Liouville problem. I. Comm. Pure Appl. Math., 36, 1983, pp. 767-783. MR720593(85d:34024)

[22] Isaacson, E., and McKean, H., and Trubowitz, E., The inverse Sturm-Liouville problem. II. Comm. Pure Appl. Math., 37, 1984, pp. 1-11. MR728263 (86f:34051)

[23] Horvath, M., On the inverse spectral theory of Schrödinger and Dirac operators, Trans. Amer. Math. Soc., 353, 2001, pp. 4155-4171. MR.1837225 (2002e:34144)

[24] Horvath, M., Inverse spectral problems and closed exponential systems, Annals of Mathematics, 162, 2005, pp. 885-918. MR.2183284 (2006k:34023)

[25] Levitan, B., On the determination of a Sturm-Liouville equation by two spectra, Amer. Math. Soc. Transl., 68, 1968, pp. 1-20.

[26] Levitan, B., Inverse Sturm-Liouville Problems, VNU Science Press, Utrecht, 1987. MR 933088 (89b:34001)

[27] Levitan, B., and Gasymov, M.G., Determination of a differential equation by two of its spectra, Russ. Math. Surv., 19, 1964, pp. 1-63. MR0162996 (29:299)

[28] Levitan, B.M., and Sargsjan, I.S., Sturm-Liouville and Dirac Operators, Kluwer, Dordrecht, 1991. MR1136037 (92i:34119)

[29] Marchenko, V., Some questions in the theory of one-dimensional linear differential operators of the second order. I, Trudy Moskov. Mat. Obsc. 1, 1952, pp. 327-420 (Russian); English transl. in Amer. Math. Soc. Transl., 2, 101, 1973, pp. 1-104. MR0058064(15:315b)

[30] Marchenko, V., Sturm-Liouville Operators and Applications, Birkhauser, Basel, 1986. MR897106 (88f:34034)

[31] McLaughlin, Joyce R., Analytical Methods for Recovering Coefficients in Differential Equations From Spectral Data, SIAM Review, 28, 1986, pp. 53-72. MR828436 (87d:34034)

[32] Psoschel, P., and Trubowitz, E., Inverse Spectral Theory, Academic Press, Boston, 1987. MR894477 (89b:34061)

[33] del rio Castillo, R., On boundary conditions of an inverse Sturm-Liouville problem, SIAM J. Appl. Math., 50, 1990, pp. 1745-1751. MR 1080519 (91j:34023)

[34] Suzuki, T., Inverse problems for heat equations on compact intervals and on circles. I, J. Math. Soc. Japan, 38, 1986, pp. 39-65. MR816222 (87f:35241) 
[35] Wei, G., and Xu, H.K., On the missing eigenvalue problem for an inverse Sturm-Liouville problem, J. Math. Pures Appl., 91, 2009, pp. 468-475. MR2517783 (2010f:34021)

[36] Zettl, A., Sturm-Liouville Theory, Surveys and Monographs 121, American Mathematical Society, 2005. MR2170950(2007a:34005)

College of Mathematics and Information Science, Shaandi Normal University, Xi'an 710062, People's Republic of China

E-mail address: weimath@vip.sina.com

Department of Applied Mathematics, National Sun Yat-Sen University, Kaohsiung 80424, TAIWAN

E-mail address: xuhk@math.nsysu.edu.tw 